\title{
On the number of principal ideals in $d$-tonal partition monoids
}

\author{
Chwas Ahmed, Paul Martin and Volodymyr Mazorchuk
}

\begin{abstract}
For a positive integer $d$, a non-negative integer $n$ and a nonnegative integer $h \leq n$, we study the number $C_{n}^{(d)}$ of principal ideals; and the number $C_{n, h}^{(\overline{d)}}$ of principal ideals generated by an element of rank $h$, in the $d$-tonal partition monoid on $n$ elements. We compute closed forms for the first family, as partial cumulative sums of known sequences. The second gives an infinite family of new integral sequences. We discuss their connections to certain integral lattices as well as to combinatorics of partitions.
\end{abstract}

Mathematics Subject Classification. 11Y55, 20M20, 05B45.

Keywords. Partition monoid, Principal ideal, Rank, Integer sequence, Hollow hexagon, Tiling.

\section{Introduction and description of the results}

Enumeration is often the starting point in understanding a given mathematical structure. Twisted monoid algebras $[6,21]$ of $d$-tonal partition monoids appear in [22] as right Schur-Weyl duals for generalized symmetric groups. These algebras are subalgebras of the classical partition algebras from $[12,13]$ and [8]. The monoids underlying the latter algebras have relatively simple principal ideal structure and well-studied representation theory, see [13,14]. The $d$-tonal subalgebras of partition algebras are more complicated. Some basics representation theory of these and related algebras was developed in [9-11] and [17]. However in the monoid case, for example, these studies cover only a trivial quotient.

The motivation for the present paper comes from our attempt to understand the structure of $d$-tonal partition algebras using combinatorics of Green's relations for the finite $d$-tonal partition monoid. The main question we answer in the present paper is what is the number of different principal 2-sided ideals 
in such a monoid. This already depends on two parameters: the difference parameter $d$ and the parameter $n$ which controls the size of our partitions. We denote the number of such ideals by $C_{n}^{(d)}$. Algebraically, there is a natural third parameter which enters the picture: the rank $h \in\{0,1, \ldots, n\}$ of the generating partition. Using this parameter, we write

$$
C_{n}^{(d)}=C_{n, 0}^{(d)}+C_{n, 1}^{(d)}+C_{n, 2}^{(d)}+\cdots+C_{n, n}^{(d)},
$$

where $C_{n, h}^{(d)}$ denotes the number of ideals generated by an element of rank $h$. We seek a closed formula for both $C_{n, h}^{(d)}$ and for $C_{n}^{(d)}$. Cases $d=1$ and $d=2$ turn out to be easy.

In Section 2, we give an alternative, purely combinatorial, definition for the numbers $C_{n}^{(d)}$ as enumerators of layers in certain graded posets. These are related to the original motivation in Section 6. The main part of the paper is devoted to the study of the case $d=3$ which occupies Section 3 .

Extra motivation for the case $d=3$ comes from its intrinsic geometricphysical interest. We give an explicit formula for $C_{n, h}^{(3)}$ in case $h$ is relatively big (i.e. $h \geq\left\lfloor\frac{n}{2}\right\rfloor$ ), see Proposition 4 , and in case $h$ is relatively small (i.e. $h \leq\left\lceil\frac{n}{3}\right\rceil$ ), see Proposition 5. The former gives a connection of our sequence to partitions with at most three parts while the latter shows a connection to triangular numbers (in fact, to a special counting of triangular numbers modulo 3). Our first main result is that the sequence $C_{n}^{(3)}$ is given by the "Cyvin sequence" (A028289 in [19]) which enumerates the number of isomorphism classes of hollow hexagons (representing polycyclic hydrocarbons), see [3,20]. In Theorem 18 of Section 4, we even give an explicit bijection between hollow hexagons and the graded poset underlying the definition of $C_{n}^{(3)}$ given in Section 2.

In Section 5, we relate our graded posets to combinatorics of partitions, and in Section 6, we make precise the connection between the combinatorially defined data discussed in the paper and the algebraic structures which motivate our investigation. Combinatorics which underlines the algebraic structure allows us to determine $C_{n}^{(d)}$ for all $d$ and $n$ in terms of partitions with at most $d$ parts, see Theorem 28 in Section 7 . As a corollary of this uniform description for all $d$, we obtain an alternative, simpler, description of A028289 using partitions with at most 3 parts.

\section{Graded posets}

\subsection{Notation and general construction}

We denote by $\mathbb{R}$ the set of all real numbers, by $\mathbb{R}_{\geq 0}$ the set of all non-negative real numbers, by $\mathbb{Z}$ the set of all integers, by $\mathbb{N}$ the set of all positive integers and by $\mathbb{Z}_{>0}$ the set of all non-negative integers.

Consider the set $\mathbb{Z}^{d}$ for some fixed $d \in \mathbb{N}$. Elements of $\mathbb{Z}^{d}$ are vectors $\mathbf{v}=\left(v_{1}, v_{2}, \ldots, v_{d}\right)$ such that $v_{i} \in \mathbb{Z}$ for all $i=1,2, \ldots, d$. The number $v_{1}+$ $v_{2}+\cdots+v_{d} \in \mathbb{Z}$ is called the height of $\mathbf{v}$ and denoted $h t(\mathbf{v})$. The set $\mathbb{Z}^{d}$ has the 
natural structure of an abelian group given by addition. The map ht : $\mathbb{Z}^{d} \rightarrow \mathbb{Z}$ is a surjective group homomorphism. For $i=1,2, \ldots, d$, we denote by $\mathbf{e}(i)$ the standard basis vector $(0,0, \ldots, 0,1,0,0, \ldots, 0)$ in $\mathbb{Z}^{d}$, in which the only non-zero element 1 stands in position $i$. Note that each $\mathbf{e}(i)$ has height 1 .

Denote by $\Lambda_{d}$ the subset $\mathbb{Z}_{\geq 0}^{d}$ in $\mathbb{Z}^{d}$. For $h \in \mathbb{Z}_{\geq 0}$, we denote by $\Lambda_{d}^{(h)}$ the set of all elements in $\Lambda_{d}$ of height $h$ and note that the set $\Lambda_{d}^{(h)}$ is finite, in fact, a standard combinatorial exercise shows that

$$
\left|\Lambda_{d}^{(h)}\right|=\left(\begin{array}{c}
h+d-1 \\
d-1
\end{array}\right) .
$$

Define $\mathbb{Z}_{(h)}^{d}=\left\{\mathbf{v} \in \mathbb{Z}^{d}: \operatorname{ht}(\mathbf{v})=h\right\}$. For a fixed subset

$$
X \subset \mathbb{Z}_{(-1)}^{d}
$$

define on $\Lambda_{d}$, the structure of a poset using the transitive closure $<_{X}$ of the following manifestly antisymmetric relation:

$$
\mathbf{v} \lessdot_{X} \mathbf{w} \quad \text { if and only if } \quad \mathbf{v}-\mathbf{w} \in X \text {. }
$$

Note from the construction that $\lessdot_{X}$ is a covering relation. Directly from the definitions, we have that $\mathbf{v} \lessdot_{X} \mathbf{w}$ implies $h t(\mathbf{v})=h t(\mathbf{w})-1$, for all $\mathbf{v}$ and w. In particular, the poset $\left(\Lambda_{d},<_{X}\right)$ is a graded poset with rank function ht $: \Lambda_{d} \rightarrow \mathbb{Z}$. Note that $X \neq X^{\prime}$ implies $\lessdot_{X} \neq \lessdot_{X^{\prime}}$.

\subsection{The poset $\mathscr{P}_{d}$}

Consider the set

$X_{d}:=\left\{\mathbf{e}(k)-\mathbf{e}(i)-\mathbf{e}(j) \in \mathbb{Z}^{d}: i, j, k \in\{1,2, \ldots, d\}\right.$ such that $\left.k \equiv i+j \bmod d\right\}$.

For example,

$$
\begin{aligned}
X_{1}= & \{(-1)\} ; \quad X_{2}=\{(-2,1),(0,-1)\} ; \\
X_{3}= & \{(1,-2,0),(-2,1,0),(-1,-1,1),(0,0,-1)\} ; \\
X_{4}= & \{(0,0,0,-1),(-1,-1,1,0),(-1,0,-1,1),(1,-1,-1,0), \\
& (-2,1,0,0),(0,-2,0,1),(0,1,-2,0)\} .
\end{aligned}
$$

Note that $<_{X_{d}}$ is defined. Denote by $\mathscr{P}_{d}$ the poset $\left(\Lambda_{d},<_{X_{d}}\right)$. Finite principal ideals of $\mathscr{P}_{d}$ are the main objects of interest in this paper. For simplicity, we will denote the relation $<_{X_{d}}$ by $\prec$. For the record, we note the following.

Lemma 1. We have $\left|X_{d}\right|=\frac{d(d-1)}{2}+1$.

Proof. The pair $\{i, j\}$ from the definition of $X_{d}$ can be chosen in $\left(\begin{array}{l}d \\ 2\end{array}\right)$ different ways for $i \neq j$ and in $d$ different ways for $i=j$. After choosing $\{i, j\}$, the element $k$ is uniquely defined. Note that the $d$ choices when $\{i, j\} \cap\{d\} \neq \varnothing$ result in the same vector $-\mathbf{e}(d)$. The claim follows.

For $\mathbf{v} \in \mathscr{P}_{d}$, we denote by $I(\mathbf{v})$ the principal ideal of $\mathscr{P}_{d}$ generated by $\mathbf{v}$, that is

$$
I(\mathbf{v}):=\{\mathbf{v}\} \bigcup\left\{\mathbf{w} \in \mathscr{P}_{d}: \mathbf{w} \prec \mathbf{v}\right\}
$$



define

For $n \in \mathbb{Z}_{\geq 0}$, we set $C_{n}^{(d)}:=|I(n \mathbf{e}(1))|$. For $h=0,1,2, \ldots, n$, we also

$$
C_{n, h}^{(d)}:=\left|I(n \mathbf{e}(1)) \cap \Lambda_{d}^{(h)}\right| .
$$

Then, we have $C_{n}^{(d)}=C_{n, 0}^{(d)}+C_{n, 1}^{(d)}+\cdots+C_{n, n}^{(d)}$. Our interest in $I(n \mathbf{e}(1))$ will be explained in Section 6 (see Theorem 27).

We observe the following structural property of $\mathscr{P}_{d}$ : for $k=1,2, \ldots, d$, consider the set $\Lambda_{d, k}$ which consists of all $\mathbf{v} \in \Lambda_{d}$ such that $v_{1}+2 v_{2}+3 v_{3}+$ $\cdots+d v_{d} \equiv k \bmod d$. Note that $\Lambda_{d, k} \cap \Lambda_{d, k^{\prime}}=\varnothing$ if $k \neq k^{\prime}$. Denote by $\mathscr{P}_{d, k}$ the poset with the underlying set $\Lambda_{d, k}$ obtained by restricting the relation $<_{X_{d}}$ to $\Lambda_{d, k}$. For $h \in \mathbb{Z}_{\geq 0}$, set $\Lambda_{d, k}^{(h)}:=\Lambda_{d, k} \cap \Lambda_{d}^{(h)}$.

\section{Proposition 2.}

(i) The poset $\mathscr{P}_{d}$ is a disjoint sum of subposets $\mathscr{P}_{d, k}$ for $k=1,2, \ldots, d$.

(ii) Each $\mathscr{P}_{d, k}$ is an indecomposable poset in the sense that it is not isomorphic to the disjoint sum of two non-empty posets.

Proof. Claim (i) follows from the definitions since $d$ divides $v_{1}+2 v_{2}+\cdots+d v_{d}$, for each $\mathbf{v} \in X_{d}$.

Note that $\mathbf{e}(k) \in \mathscr{P}_{d, k}$. Therefore, to prove claim (ii), it is enough to show that $\mathbf{e}(k) \prec \mathbf{v}$, for any $\mathbf{v} \in \mathscr{P}_{d, k}$ of height at least 2 . However, if $\mathbf{v}$ has height at least 2 , then either $\mathbf{v}$ has a coordinate which is greater than or equal to 2 , or $\mathbf{v}$ has at least two non-zero coordinates. Therefore, there is $\mathbf{x} \in X_{d}$ such that $\mathbf{v}+\mathbf{x} \in \Lambda_{d}$. We have $\mathbf{v}+\mathbf{x} \prec \mathbf{v}$ and from the observation in the previous paragraph we see that $\mathbf{v}+\mathbf{x} \in \Lambda_{d, k}$. Therefore $\mathbf{e}(k) \prec \mathbf{v}$ follows by induction on the height of $\mathbf{v}$. This completes the proof.

From the above proof, it follows that for $k \neq d$, the element $\mathbf{e}(k)$ is the minimum element in $\mathscr{P}_{d, k}$ and that the minimum element in $\mathscr{P}_{d, d}$ is $\mathbf{0}:=(0,0, \ldots, 0)$.

\subsection{The case $d=1$}

In the case $d=1$, the map

$$
\begin{aligned}
& \mathscr{P}_{1} \rightarrow\left(\mathbb{Z}_{\geq 0},<\right) \\
& (i) \mapsto \quad i
\end{aligned}
$$

is an isomorphism of posets. For $n \in \mathbb{Z}_{\geq 0}$, we have

$$
I(n \mathbf{e}(1))=\{(0),(1),(2), \ldots,(n)\}
$$

and thus $C_{n}^{(1)}=n+1$. Note that in this case, the poset $\mathscr{P}_{1}=\mathscr{P}_{1,1}$ is indecomposable.

\subsection{The case $d=2$}

Our first observation in this case is that the maps

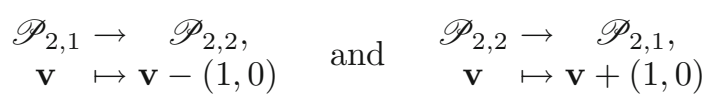




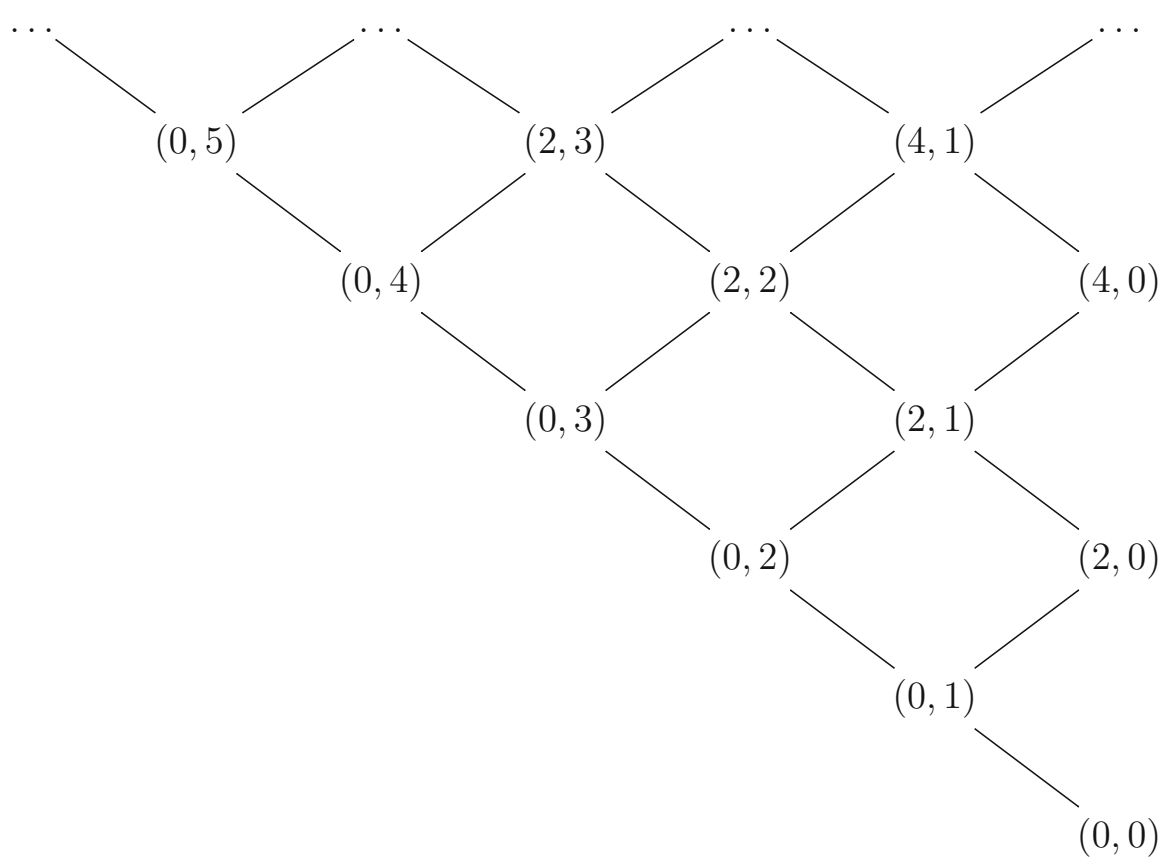

Figure 1. Hasse diagram for $\mathscr{P}_{2,2}$

are mutually inverse isomorphisms of posets. Consequently, we have $C_{n}^{(2)}=$ $C_{n+1}^{(2)}$, for all even $n \in \mathbb{Z}_{\geq 0}$. The lower part of the Hasse diagram for $\mathscr{P}_{2,2}$ is shown in Figure 1.

It follows immediately that for $k \in \mathbb{Z}_{\geq 0}$, we have

$$
C_{2 k}^{(2)}=\frac{(k+1)(k+2)}{2} .
$$

We also note that $I(2 k \mathbf{e}(1)) \subset I(2(k+1) \mathbf{e}(1))$, for all $k \in \mathbb{Z}_{\geq 0}$, and that

$$
\bigcup_{k \in \mathbb{Z}_{\geq 0}} I(2 k \mathbf{e}(1))=\mathscr{P}_{2,2} .
$$

It is also worth pointing out that for each $k \in \mathbb{Z}_{\geq 0}$, the poset $I(2 k \mathbf{e}(1))$ is isomorphic to the poset $I(2 k \mathbf{e}(1))^{\text {op }}$ (the latter is obtained from $I(2 k \mathbf{e}(1))$ by reversing the partial order).

\section{The case $d=3$}

As we will show below, the case $d=3$ has several interesting connections to integral sequences. Our study of this case is the main part of the present paper. 


\subsection{Isomorphism of $\mathscr{P}_{3,1}$ and $\mathscr{P}_{3,2}$}

The symmetric group $S_{2}$ acts on $\Lambda_{3}$ as follows: for $\mathbf{v}=\left(v_{1}, v_{2}, v_{3}\right)$ and $\pi \in S_{2}$ we have $\pi \cdot \mathbf{v}=\left(v_{\pi(1)}, v_{\pi(2)}, v_{3}\right)$. Note that the set $X_{3}$ (which can be found in Subsection 2.2) is invariant with respect to the action of $S_{2}$. Therefore, this action induces an action on $\mathscr{P}_{3}$ by automorphisms. Using this action, we can swap e(1) and e(2) and hence $\mathscr{P}_{3,1}$ and $\mathscr{P}_{3,2}$ (cf. proof of Proposition 2). Therefore, the posets $\mathscr{P}_{3,1}$ and $\mathscr{P}_{3,2}$ are isomorphic.

\subsection{An alternative description}

In this subsection, we observe that $\mathscr{P}_{3}$ can be defined by restriction from $\mathbb{Z}^{3}$. This is a useful property for computations using computers.

We mimic the definition of $\mathscr{P}_{3}$ starting from $\mathbb{Z}^{3}$ instead of $\Lambda_{3}$. Consider the set $X_{3}$ as defined in Subsection 2.2. Use (2.2) to define the covering relation on $\mathbb{Z}^{3}$ and let $\prec^{\prime}$ denote the partial order on $\mathbb{Z}^{3}$ induced by this covering relation. Our main observation here is the following:

Proposition 3. The relation $\prec$ coincides with the restriction of the relation $\prec^{\prime}$ to $\Lambda_{3}$.

Proof. Let $\preceq^{\prime}$ denote the restriction of the relation $\prec^{\prime}$ to $\Lambda_{3}$. Clearly, $\prec \subset \preceq^{\prime}$, so we only need to show that $\prec^{\prime} \subset \prec$.

Let $\mathbf{v}, \mathbf{w} \in \Lambda_{3}$ be such that $\mathbf{v} \prec^{\prime} \mathbf{w}$. We have to show that $\mathbf{v} \prec \mathbf{w}$. Assume that this is not the case and that the pair $(\mathbf{v}, \mathbf{w})$ satisfying $\mathbf{v} \prec^{\prime} \mathbf{w}$ and $\mathbf{v} \nprec \mathbf{w}$ is chosen such that $\mathrm{ht}(\mathbf{w}-\mathbf{v})=k \in \mathbb{N}$ is minimal possible. As $\mathbf{v} \prec^{\prime} \mathbf{w}$, there is a sequence of elements $\mathbf{x}_{1}, \mathbf{x}_{2}, \ldots, \mathbf{x}_{k} \in X_{3}$ such that

$$
\mathbf{v}=\mathbf{w}+\mathbf{x}_{1}+\mathbf{x}_{2}+\cdots+\mathbf{x}_{k}
$$

Consider $\mathbf{v}_{i}=\mathbf{v}-\mathbf{x}_{i}$ for $i=1,2, \ldots, k$. We claim that all $\mathbf{v}_{i} \notin \Lambda_{3}$. Indeed, if $\mathbf{v}_{i} \in \Lambda_{3}$, then we would have $\mathbf{v} \prec \mathbf{v}_{i}$ and $\mathbf{v}_{i} \prec^{\prime} \mathbf{w}$. This would imply $\mathbf{v}_{i} \nprec \mathbf{w}$ which would contradict our minimal choice of $k$. In particular, none of the $\mathbf{x}_{i}$ 's equals $(0,0,-1)$ since $\mathbf{v}-(0,0,-1) \in \Lambda_{3}$ because $\mathbf{v} \in \Lambda_{3}$.

The next step is to show that none of the $\mathbf{x}_{i}$ 's equals $(-1,-1,1)$. Otherwise, without loss of generality, we may assume that $\mathbf{x}_{k}=(-1,-1,1)$. Then, we have $\mathbf{v}_{k} \notin \Lambda_{3}$, and hence, $\mathbf{v}=(*, *, 0)$ and $\mathbf{v}_{k}=(*, *,-1)$. Furthermore, we have

$$
(*, *,-1)=\mathbf{v}_{k}=\mathbf{w}+\mathbf{x}_{1}+\mathbf{x}_{2}+\cdots+\mathbf{x}_{k-1} .
$$

Since $\mathbf{w} \in \Lambda_{3}$, the third coordinate in $\mathbf{w}$ is non-negative. This means that at least one of the $\mathbf{x}_{i}$ 's must have negative third coordinate. The only element in $X_{3}$ with negative third coordinate is $(0,0,-1)$. However, in the previous paragraph, we already established that none of the $\mathbf{x}_{i}$ 's equals $(0,0,-1)$, a contradiction.

Therefore, each $\mathbf{x}_{i}$ is equal to either $(-2,1,0)$ or $(1,-2,0)$. Assume that all $\mathbf{x}_{i}$ are equal, say to $(-2,1,0)$ (the case of $(1,-2,0)$ is similar). Then, $\mathbf{v}=$ $\mathbf{w}+k(-2,1,0)$. Since both $\mathbf{v}$ and $\mathbf{w}$ are in $\Lambda_{3}$, we have $\mathbf{w}+i(-2,1,0) \in \Lambda_{3}$ for all $i$ such that $1 \leq i \leq k$. Therefore, $\mathbf{v} \prec \mathbf{w}$, a contradiction. 


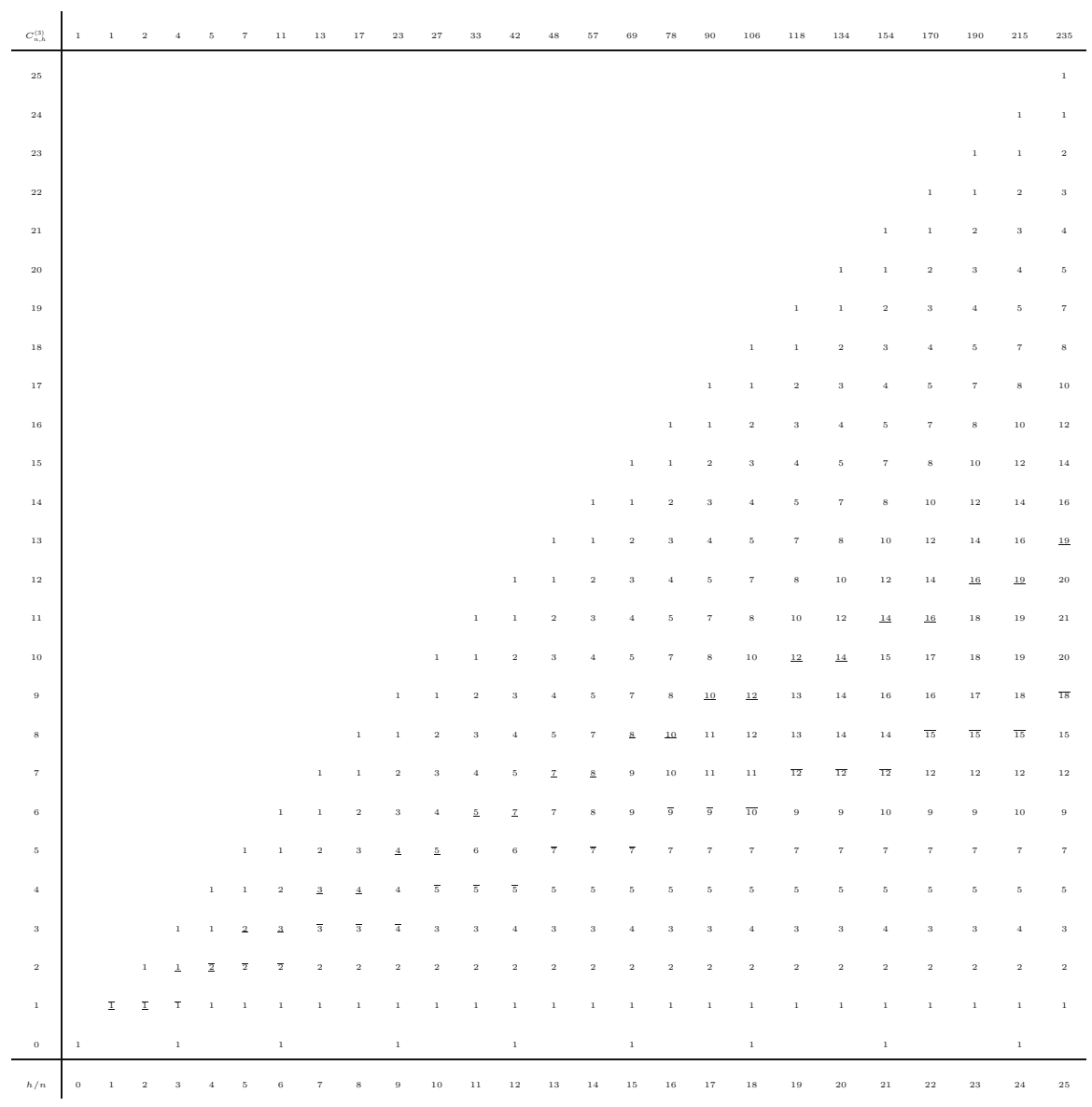

Figure 2. Values of $C_{n, h}^{(3)}$ for $n \leq 25$

The last paragraph establishes that at least one of the $\mathbf{x}_{i}$ 's equals $(-2,1,0)$ and at least one equals $(1,-2,0)$. This implies $\mathbf{v}-(-2,1,0)-(1,-2,0)=$ $\mathbf{v}+(1,1,0) \prec^{\prime} \mathbf{w}$. At the same time, we have

$$
\mathbf{v}+(0,0,1), \mathbf{v}+(1,1,0) \in \Lambda_{3}
$$

as $\mathbf{v} \in \Lambda_{3}$ and

$$
\mathbf{v} \prec \mathbf{v}+(0,0,1) \prec \mathbf{v}+(0,0,1)+(1,1,-1)=\mathbf{v}+(1,1,0) .
$$

This implies $\mathbf{v}+(1,1,0) \nprec \mathbf{w}$ which again contradicts our minimal choice of $k$. The claim follows.

\subsection{Small values}

The table of $C_{n, h}^{(3)}$ for small values of $n$ is given in Figure 2 (computed first by hands, up to $n=15$, and then checked and extended using Proposition 3 and MAPLE). Please ignore the underlines and the overlines for the moment. 


\subsection{Values of $C_{n, h}^{(3)}$ for large $h$}

The sequence $A 001399(n)$ in [19] lists the number of partitions of $n$ into at most 3 parts. Here are the first 25 elements in this sequence:

$$
1,1,2,3,4,5,7,8,10,12,14,16,19,21,24, \ldots
$$

Comparison with columns of Figure 2 suggests that the upper part of each column in Figure 2 bounded by the underlined element is an initial segment of A001399. Indeed, we have the following claim:

Proposition 4. For $h \geq\left\lceil\frac{n}{2}\right\rceil$, we have $C_{n, h}^{(3)}=A 001399(n-h)$.

Proof. Let $(a, b, c)$ be a partition of $n-h$ in at most three parts, that is, $a, b, c \in \mathbb{Z}_{\geq 0}, a \geq b \geq c$ and $a+b+c=n-h$. Then, we claim that

$$
\mathbf{v}_{(a, b, c)}:=(n, 0,0)+a(-2,1,0)+b(-1,-1,1)+c(0,0,-1) \prec(n, 0,0) .
$$

By Proposition 3, it is enough to show that $\mathbf{v}_{(a, b, c)} \in \Lambda_{3}$. The latter however follows from $2 a+b \leq n$ (thanks to $h \geq\left\lceil\frac{n}{2}\right\rceil$ ) and $b \geq c$ (thanks to the fact that $(a, b, c)$ is a partition).

The vectors $(-2,1,0),(-1,-1,1)$ and $(0,0,-1)$ are linearly independent, which implies that $\mathbf{v}_{(a, b, c)} \neq \mathbf{v}_{\left(a^{\prime}, b^{\prime}, c^{\prime}\right)}$ provided that $(a, b, c) \neq\left(a^{\prime}, b^{\prime}, c^{\prime}\right)$. Therefore, $C_{n, h}^{(3)} \geq A 001399(n-h)$.

Now, consider some $v \in I(n \mathbf{e}(1))$ with height $h$. Then,

$$
v=(n, 0,0)+a(-2,1,0)+b(-1,-1,1)+c(0,0,-1)+d(1,-2,0),
$$

for some $a, b, c, d \in \mathbb{Z}_{\geq 0}$. Since the second coordinate of $v$ is non-negative, we have $a \geq d$. Since

$$
(1,-2,0)=-(-2,1,0)+(-1,-1,1)+(0,0,-1),
$$

we have

$$
v=(n, 0,0)+(a-d)(-2,1,0)+(b+d)(-1,-1,1)+(c+d)(0,0,-1)
$$

and thus may assume that $d=0$ in (3.1). We have $a+b+c=n-h$ since $v$ has height $h, b \geq c$ as the third coordinate of $v$ is non-negative and $a \geq b$ as the second coordinate of $v$ is non-negative. Therefore, $v=\mathbf{v}_{(a, b, c)}$, for the partition $(a, b, c)$ of $n-h$. The claim of the proposition follows.

\subsection{Values of $C_{n, h}^{(3)}$ for small $h$}

We start this subsection with the following observation:

Proposition 5. For $h \leq\left\lceil\frac{n}{3}\right\rceil$, we have $\Lambda_{3}^{(h)} \cap I(n \mathbf{e}(1))=\Lambda_{3}^{(h)} \cap \Lambda_{3, k}$, where $k \in\{1,2,3\}$ is such that $n \equiv k(\bmod 3)$.

Proof. As $I(n \mathbf{e}(1)) \subset \Lambda_{3, k}$, for our choice of $k$, to prove the assertion of this proposition, we only need to show that $\left(\Lambda_{3}^{(h)} \cap \Lambda_{3, k}\right) \subset\left(\Lambda_{3}^{(h)} \cap I(n \mathbf{e}(1))\right)$. If $v \in\left(\Lambda_{3}^{(h)} \cap \Lambda_{3, k}\right) \backslash\left(\Lambda_{3}^{(h)} \cap I(n \mathbf{e}(1))\right)$, for some $h$, then $v+(0,0,1) \in\left(\Lambda_{3}^{(h+1)} \cap\right.$ $\left.\Lambda_{3, k}\right) \backslash\left(\Lambda_{3}^{(h+1)} \cap I(n \mathbf{e}(1))\right)$ since $\left.I(n \mathbf{e}(1))\right)$ is an ideal. Therefore, it is enough to prove the proposition for $h=\left\lceil\frac{n}{3}\right\rceil$ which we from now on assume. Set $q:=\left\lfloor\frac{n}{3}\right\rfloor$. We will have to consider three different cases depending on $k$. 


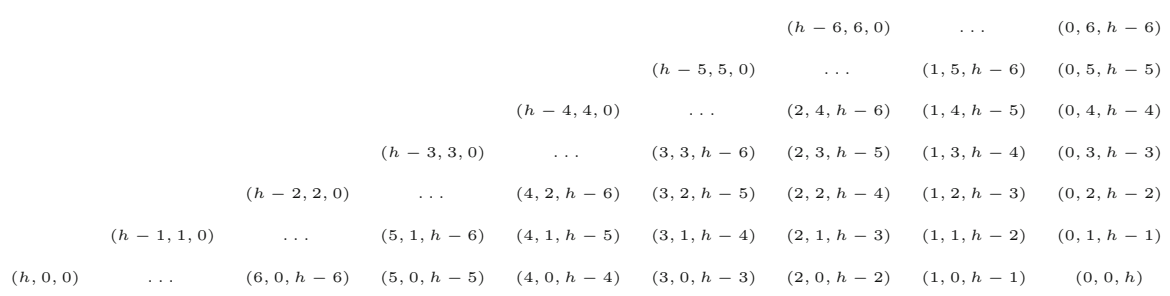

FigURE 3. Triangular arrangement of $\Lambda_{3}^{(h)}$

Case 1: $k=3$. In this case, $q=h=\frac{n}{3}$. Let $(a, b, c) \in \Lambda_{3}^{(h)} \cap \Lambda_{3, k}$, that is $a, b, c \in \mathbb{Z}_{\geq 0}, a+b+c=h$ and 3 divides $a+2 b$. In this case, we have

$$
(n-3 c-2 b, b, c)=(n, 0,0)+(b+c)(-2,1,0)+c(-1,-1,1) \prec(n, 0,0) .
$$

Now, $n-3 c-2 b=3 a+b$. Since 3 divides both $a+2 b$ and $3 a+3 b$, it also divides $2 a+b$. Therefore, there is $p \in \mathbb{Z}_{\geq 0}$ such that $2 a+b=3 p$. We have

$$
\begin{aligned}
& (a, b, c)=(n-3 c-2 b-3 p, b, c)= \\
& =(n-3 c-2 b, b, c)+p(-2,1,0)+p(-1,-1,1)+p(0,0,-1) \\
& \quad \prec(n-3 c-2 b, b, c) .
\end{aligned}
$$

Combining (3.2) and (3.3) implies $(a, b, c) \prec(n, 0,0)$ and hence $(a, b, c) \in$ $I(n \mathbf{e}(1))$.

Case 2: $k=2$. In this case, $q=h-1$ and $n=3 h-1$. Let $(a, b, c) \in$ $\Lambda_{3}^{(h)} \cap \Lambda_{3, k}$, that is $a, b, c \in \mathbb{Z}_{\geq 0}, a+b+c=h$ and 3 divides $a+2 b-2$. Formula (3.2) still holds in this case. Now, $n-3 c-2 b=3 a+b-1$. Since 3 divides both $a+2 b-2$ and $3 a+3 b$, it also divides $2 a+b-1$. Therefore, there is $p \in \mathbb{Z}_{>0}$ such that $2 a+b-1=3 p$. Formula (3.3) still holds in this case. Again it follows that $(a, b, c) \in I(n \mathbf{e}(1))$.

Case 3: $k=1$. In this case, $q=h-1$ and $n=3 h-2$. Let $(a, b, c) \in$ $\Lambda_{3}^{(h)} \cap \Lambda_{3, k}$, that is $a, b, c \in \mathbb{Z}_{\geq 0}, a+b+c=h$ and 3 divides $a+2 b-1$. Formula (3.2) still holds in this case. Now, $n-3 c-2 b=3 a+b-2$. Since 3 divides both $a+2 b-1$ and $3 a+3 b$, it also divides $2 a+b-2$. Therefore, there is $p \in \mathbb{Z}_{\geq 0}$ such that $2 a+b-2=3 p$. Formula (3.3) still holds in this case, and we similarly obtain $(a, b, c) \in I(n \mathbf{e}(1))$.

\section{Lemma 6.}

(i) If 3 does not divide $h$, then $\left|\Lambda_{3,1}^{(h)}\right|=\left|\Lambda_{3,2}^{(h)}\right|=\left|\Lambda_{3,3}^{(h)}\right|$.

(ii) If 3 divides $h$, then $\left|\Lambda_{3,1}^{(h)}\right|=\left|\Lambda_{3,2}^{(h)}\right|=\left|\Lambda_{3,3}^{(h)}\right|-1$.

Proof. We prove both statements at the same time by induction on $h$. Let us arrange elements of $\Lambda_{3}^{(h)}$ in a triangular array as shown in Figure 3 . 
Writing down the residue modulo 3 of the expression $a+2 b$ for each element $(a, b, c)$ in Figure 3, we get

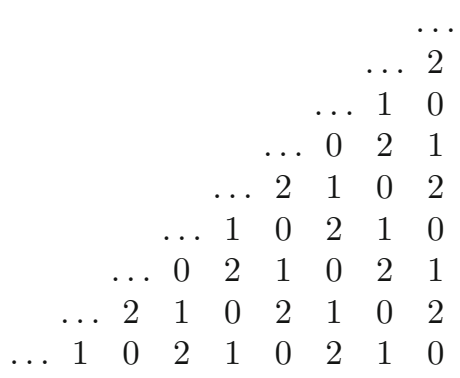

For a fixed $h$, the set $\Lambda_{3}^{(h)}$ corresponds to the first $h+1$ "bottom-left-to-topright" diagonals starting from the bottom right corner. The induction step $h \rightarrow h+1$ corresponds to adding the next diagonal.

By a direct inspection of the above tables, we have

$$
\begin{aligned}
& 0=\left|\Lambda_{3,1}^{(0)}\right|=\left|\Lambda_{3,2}^{(0)}\right|=\left|\Lambda_{3,3}^{(0)}\right|-1, \\
& 1=\left|\Lambda_{3,1}^{(1)}\right|=\left|\Lambda_{3,2}^{(1)}\right|=\left|\Lambda_{3,3}^{(1)}\right|, \\
& 2=\left|\Lambda_{3,1}^{(2)}\right|=\left|\Lambda_{3,2}^{(2)}\right|=\left|\Lambda_{3,3}^{(2)}\right|,
\end{aligned}
$$

which establishes the basis of our induction.

Note that the residues in each diagonal follow a cyclic order on $0,1,2$ (as one step up along the diagonal decreases the first coordinate by 1 and increases the second coordinate by 1 , thus changing $a+2 b$ to $(a-1)+2(b+1))$. In particular, if the number of elements on a new diagonal is divisible by 3 , it contains the same number of 0's, 1's and 2's. This proves the induction step in the case when 3 divides $h-1$.

If 3 divides $h-2$, then the new diagonal contains an extra zero compared to the common number of 1's and 2's. If 3 divides $h$, then the new diagonal contains one zero less than the common number of 1's and 2's. Put together this implies the induction step and completes the proof of the proposition.

For a set $X$, we denote by $\delta_{X}$ the indicator function of $X$, that is,

$$
\delta_{X}(x)= \begin{cases}1, & x \in X ; \\ 0, & x \notin X .\end{cases}
$$

Corollary 7. For $h \leq\left\lceil\frac{n}{3}\right\rceil$, we have

$$
C_{n, h}^{(3)}=\frac{(h+1)(h+2)+\left(6 \delta_{3 \mathbb{Z}}(n)-2\right) \delta_{3 \mathbb{Z}}(h)}{6} .
$$

Proof. We have $C_{n, h}^{(3)}=\left|I(n \mathbf{e}(1)) \cap \Lambda_{3}^{(h)}\right|$ by definition and $\left|I(n \mathbf{e}(1)) \cap \Lambda_{3}^{(h)}\right|=$ $\left|\Lambda_{3}^{(h)} \cap \Lambda_{3, k}\right|$ by Proposition 5. Further, $\left|\Lambda_{3}^{(h)} \cap \Lambda_{3, k}\right|=\left|\Lambda_{3, k}^{(h)}\right|$ again by definition. Now the claim follows applying Lemma 6 and Formula (2.1) and keeping $\left|\Lambda_{3}^{(h)}\right|=\left|\Lambda_{3,1}^{(h)}\right|+\left|\Lambda_{3,2}^{(h)}\right|+\left|\Lambda_{3,3}^{(h)}\right|$ in mind. 
In the case when 3 does not divide $n$, the sequence $\frac{(h+1)(h+2)-2 \delta_{3 Z}(h)}{6}$ is $A 001840(h)$ from [19]. In the case when 3 divides $n$, the sequence $\frac{(h+1)(h+2)+4 \delta_{3 \mathbb{Z}}(h)}{6}$ is $A 007997(h+2)$ from [19]. However, it seems that our interpretation of both these sequences does not appear on [19] at the moment. We note that the sequence $A 001840(h+1)-A 001840(h)$ is the sequence

$$
1,1,1,2,2,2,3,3,3,4,4,4,5,5,5, \ldots,
$$

while the sequence $A 007997(h+3)-A 007997(h+2)$ is the sequence

$$
1,0,1,2,1,2,3,2,3,4,3,4,5,4,5,6,5,6, \ldots
$$

The latter sequence should be compared with the fourth sequence which will be constructed in Subsection 3.6 below.

Each column in Figure 2 contains a unique overlined element. This element corresponds to the upper bound $\left\lceil\frac{n}{3}\right\rceil$ for the value of $h$ for which $C_{n, h}^{(3)}$ is given by Corollary 7. In other words, this element and all elements below it in the same column are given by an initial segment of $A 007997(h+2)$ or $A 001840(h)$, if 3 does or does not divide $n$, respectively.

Problem 8. Find a closed formula for $C_{n, h}^{(3)}$, where $\left\lceil\frac{n}{3}\right\rceil<h<\left\lceil\frac{n}{2}\right\rceil$.

We do not know how hard this problem is, we do not see how to approach it in full generality.

\subsection{Sequence $A 028289$}

The sequence A028289 in [19] lists coefficients in the expansion of $\frac{1+t^{2}+t^{3}+t^{5}}{(1-t)\left(1-t^{3}\right)\left(1-t^{4}\right)\left(1-t^{6}\right)}$. Here are the first 25 elements in this sequence:

$1,1,2,4,5,7,11,13,17,23,27,33,42,48,57,69,78,90,106,118,134,154$, $170,190,215,235, \ldots$

This sequence appears in [3]. Comparison with the first row of Figure 2 suggests that $C_{n}^{(3)}=A 028289(n)$ for all $n$. We will prove this in the next subsection. In this subsection, we propose two constructions of $A 028289$, alternative to its definition on [19]. The first construction consists of five combinatorial steps.

- Consider first the sequence $0,1,2,3,4,5 \ldots$ of all non-negative integers.

- Construct the second sequence $0,1,1,2,2,3,3,4,4,5,5, \ldots$ by repeating all non-zero terms in the previous sequence twice.

- Define the third sequence as the sequence of partial sums of the second sequence: $0,1,2,4,6,9,12,16,20,25,30, \ldots$.

- Construction of the fourth sequence is the most complicated one. The sequence is:

$1,0,1,2,1,2,4,2,4,6,4,6,9,6,9,12,9,12,16,12,16,20,16,20, \ldots$

and this is obtained by adding appropriately shifted five-term frames of the form $(i, 0, i, 0, i)$, where $i$ an element of the third sequence, as shown 
here:

$$
\begin{aligned}
& 10101 \\
& 20202 \\
& 40404 \\
& \begin{array}{lllll}
6 & 0 & 6 & 0 & 6
\end{array} \\
& \text { add: } 101212424646 \ldots \ldots \ldots
\end{aligned}
$$

- The final, fifth, sequence is the sequence of partial sums of the fourth sequence:

$1,1,2,4,5,7,11,13,17,23,27,33,42,48,57,69,78,90,106,118,134,154,170,190, \ldots$

Proposition 9. The fifth sequence constructed above coincides with A028289.

Proof. Let us compute the generating function of all sequences constructed above. For the first sequence, the generating function is

$$
f(t):=\frac{t}{(1-t)^{2}}
$$

For the second sequence, we get

$$
f\left(t^{2}\right)+\frac{1}{t} f\left(t^{2}\right)=\frac{t+t^{2}}{\left(1-t^{2}\right)^{2}} .
$$

Convolution with $1,1,1, \ldots$, that is, the sequence with generating function $\frac{1}{1-t}$, implies that the generating function for the third sequence is

$$
g(t)=\frac{t+t^{2}}{(1-t)\left(1-t^{2}\right)^{2}}
$$

The generating function for the fourth sequence is

$$
\frac{g\left(t^{3}\right)}{t^{3}}+\frac{t^{2} g\left(t^{3}\right)}{t^{3}}+\frac{t^{4} g\left(t^{3}\right)}{t^{3}}=\frac{\left(1+t^{3}\right)\left(1+t^{2}+t^{4}\right)}{\left(1-t^{6}\right)^{2}\left(1-t^{3}\right)}=\frac{1+t^{2}+t^{4}}{\left(1-t^{6}\right)\left(1-t^{3}\right)^{2}}
$$

Finally, yet another convolution with $1,1,1, \ldots$ gives the generating function

$$
\frac{1+t^{2}+t^{4}}{(1-t)\left(1-t^{6}\right)\left(1-t^{3}\right)^{2}}
$$

for the fifth sequence. The latter generating function coincides with the generating function

$$
\frac{1+t^{2}+t^{3}+t^{5}}{(1-t)\left(1-t^{3}\right)\left(1-t^{4}\right)\left(1-t^{6}\right)}
$$

of $A 028289$ since

$$
\left(1+t^{2}+t^{4}\right)\left(1-t^{4}\right)=1+t^{2}-t^{6}-t^{8}=\left(1+t^{2}+t^{3}+t^{5}\right)\left(1-t^{3}\right) .
$$

The claim follows.

Our second construction of $A 028289$ (which is relevant for Theorem 11 in the following subsection) uses the following observation: 
Lemma 10. We have

$$
\frac{1+t^{2}+t^{3}+t^{5}}{(1-t)\left(1-t^{3}\right)\left(1-t^{4}\right)\left(1-t^{6}\right)}=\frac{1}{1-t} \cdot \frac{1}{1-t^{3}} \cdot\left(1+t^{2}+t^{3}+t^{4}+t^{5}+t^{7}\right) \cdot \frac{1}{\left(1-t^{6}\right)^{2}} .
$$

Proof. We have to check that

$$
\frac{1+t^{2}+t^{3}+t^{5}}{1-t^{4}}=\frac{1+t^{2}+t^{3}+t^{4}+t^{5}+t^{7}}{1-t^{6}} .
$$

This is a straightforward computation.

Lemma 10 implies that $A 028289$ can be constructed in the following four combinatorial steps.

- Consider first the sequence $1,2,3,4,5 \ldots$ of all positive integers.

- Construct the second sequence $1,0,1,1,1,1,2,1,2,2,2,2,3,2, \ldots$ by repeating the pattern $i, *, i, i, i, i, *, i$ of the terms in the previous sequence using shift in six positions.

- Construct the third sequence $1,0,1,2,1,2,4,2,4, \ldots$ by convolution of the second sequence with $1,0,0,1,0,0,1,0,0,1, \ldots$.

- The final, fourth, sequence is the sequence of partial sums of the third sequence:

$1,1,2,4,5,7,11,13,17,23,27,33,42,48,57,69,78,90,106,118,134,154,170,190, \ldots$

\subsection{Computation of $C_{n}^{(3)}$}

Here, we prove our first main result.

Theorem 11. We have $C_{n}^{(3)}=A 028289(n)$ for all $n \in \mathbb{Z}_{\geq 0}$.

Proof. Taking Lemma 10 into account, to prove the assertion of our theorem, it is enough to show that

$$
\sum_{n \geq 0} C_{n}^{(3)} t^{n}=\frac{1}{1-t} \cdot \frac{1}{1-t^{3}} \cdot\left(1+t^{2}+t^{3}+t^{4}+t^{5}+t^{7}\right) \cdot \frac{1}{\left(1-t^{6}\right)^{2}} .
$$

For a variable $n$, consider the sets

$$
\begin{aligned}
\tilde{D}_{n} & :=(n, 0,0)+\mathbb{Z}(-2,1,0)+\mathbb{Z}(-1,-1,1)+\mathbb{Z}(0,0,-1), \\
D_{n} & :=(n, 0,0)+\mathbb{Z}(-2,1,0)+\mathbb{Z}(-3,0,1) .
\end{aligned}
$$

For $v \in \tilde{D}_{n}$, we denote by $\Phi(v)=\Phi_{n}(v)$ the unique element in $D_{n}$ for which we have $\Phi(v)-v \in \mathbb{Z}(-3,0,0)$. This is well-defined as the $\mathbb{Z}$-linear span of the linearly independent vectors $(-2,1,0),(-1,-1,0)$ and $(0,0,1)$ coincides with the $\mathbb{Z}$-linear span of the linearly independent vectors $(-2,1,0),(-3,0,1)$ and $(-3,0,0)$.

Our first observation is the following:

Lemma 12. For any $\mathbf{v} \in I(n \mathbf{e}(1))$, we have

$$
\Phi(\mathbf{v}) \in(n, 0,0)+\mathbb{Z}_{\geq 0}(-2,1,0)+\mathbb{Z}_{\geq 0}(-3,0,1) .
$$


Proof. We have

$$
\mathbf{v}=(n, 0,0)+a(-2,1,0)+b(1,-2,0)+c(-1,-1,1)+d(0,0,-1),
$$

for $a, b, c, d, \in \mathbb{Z}_{\geq 0}$, by definition. From $\mathbf{v} \in I(n \mathbf{e}(1))$, by looking at the second and the third coordinates of $\mathbf{v}$, we obtain $a-2 b-c \geq 0$ and $c-d \geq 0$. Now we rewrite

$$
\mathbf{v}=(n, 0,0)+(a-2 b-c)(-2,1,0)+(c-d)(-3,0,1)+(b+d)(-3,0,0) .
$$

As $(n, 0,0)+(a-2 b-c)(-2,1,0)+(c-d)(-3,0,1) \in D_{n}$, the claim of the lemma follows from the definition of $\Phi$.

Motivated by Lemma 12 , for $i \in \mathbb{Z}_{\geq 0}$, set $f_{i}:=\left|T_{i}\right|$, where

$$
T_{i}:=\left\{\mathbf{v} \in(n, 0,0)+\mathbb{Z}_{\geq 0}(-2,1,0)+\mathbb{Z}_{\geq 0}(-3,0,1): \mathbf{v}=(n-i, *, *)\right\} .
$$

Lemma 13. We have

$$
\sum_{i \geq 0} f_{i} t^{i}=\frac{1+t^{2}+t^{3}+t^{4}+t^{5}+t^{7}}{\left(1-t^{6}\right)^{2}} .
$$

Proof. The proof is illustrated in Figure 4. By definition, $f_{i}$ enumerates lattice points of the two-dimensional cone $\mathbf{C}:=(n, 0,0)+\mathbb{Z}_{\geq 0}(-2,1,0)+\mathbb{Z}_{\geq 0}(-3,0,1)$ (the points of $\mathbf{C}$ are depicted as bullet points in Figure 4) belonging to the line, in the plane determined by the cone, given by the condition that the first coordinate of the point on the line equals $n-i$ (these lines are depicted as dashed lines in Figure 4).

A direct calculation gives the following values for small $i$ :

$$
\begin{array}{l|llllllll}
i & 0 & 1 & 2 & 3 & 4 & 5 & 6 & 7 \\
\hline f_{i} & 1 & 0 & 1 & 1 & 1 & 1 & 2 & 1
\end{array}
$$

For $i=6$, we, for the first time, have $f_{i}=2>1$. This implies that $f_{i}$ satisfies the recursion $f_{i+6}=f_{i}+1$, for all $i \geq 0$. Indeed, from Figure 4 , we see that $\mathbf{C}$ is the disjoint union of the shifted copy $\mathbf{C}+(-6,0,2)$ of $\mathbf{C}$ and the remaining strip of width 2 going north-east in Figure 4. The recursion follows by noting that for $i \neq 1$, the corresponding dashed line intersects the remaining strip in exactly one point and that for $i \geq 6$, the intersection of the corresponding dashed line with $\mathbf{C}+(-6,0,2)$ has exactly $f_{i-6}$ points, due to the observation above and the linearity of the definitions.

The claim of the lemma follows by combining the recursion $f_{i+6}=f_{i}+1$ with the initial values listed in (3.5).

For each $n \in \mathbb{Z}_{>0}$, mapping $\mathbf{v} \mapsto \mathbf{v}+(1,0,0)$ defines an injection from $I((n-1) \mathbf{e}(1))$ to $I(n \mathbf{e}(1))$. Set $g_{n}:=C_{n}^{(3)}-C_{n-1}^{(3)} \geq 0$ (under the convention $\left.C_{-1}^{(3)}=0\right)$. Then, we have

$$
C_{n}^{(3)}=g_{n}+g_{n-1}+\cdots+g_{0}
$$

by construction. This reduces the claim of the theorem to the following crucial observation: 


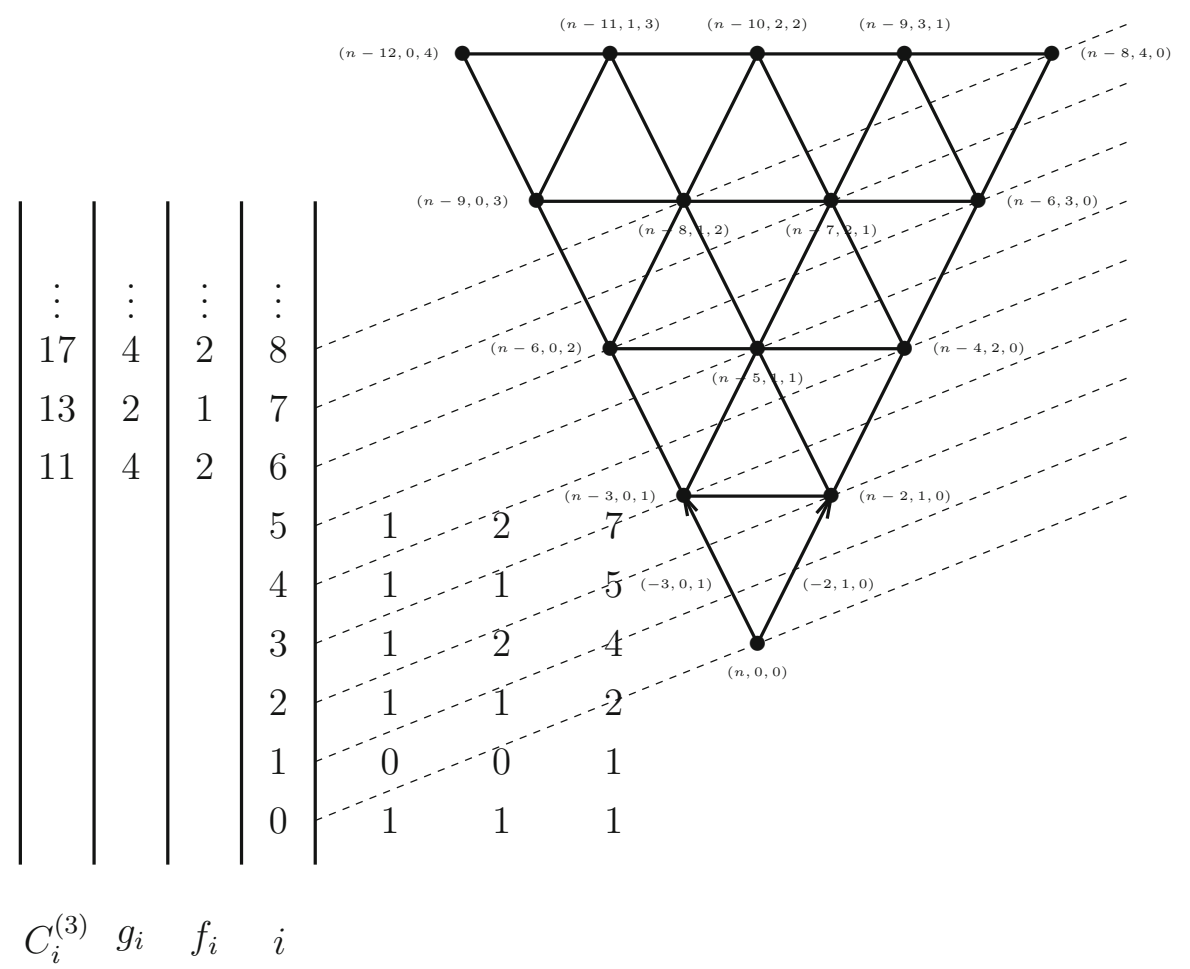

FiguRE 4. Geometric illustration of the proof of Lemma 13 and Theorem 11

Lemma 14. We have $g_{n}=f_{n}+f_{n-3}+f_{n-6}+\ldots$, for all $n$, where we assume $f_{k}=0$, for $k<0$.

Proof. First of all, we claim that the map

$$
\begin{aligned}
I((n-1) \mathbf{e}(1)) & \rightarrow I(n \mathbf{e}(1)) \\
\mathbf{v} & \mapsto \mathbf{v}+(1,0,0)
\end{aligned}
$$

induces a bijection between $I((n-1) \mathbf{e}(1))$ and the set

$$
\left\{\mathbf{v}=\left(v_{1}, v_{2}, v_{3}\right) \in I(n \mathbf{e}(1)): v_{1} \neq 0\right\} .
$$

Indeed, the inverse map is easily seen to be given by $\mathbf{w} \mapsto \mathbf{w}-(1,0,0)$.

Therefore, we need to show that $f_{n}+f_{n-3}+f_{n-6}+\ldots$ enumerates the set

$$
R:=\left\{\mathbf{v}=\left(v_{1}, v_{2}, v_{3}\right) \in I(n \mathbf{e}(1)): v_{1}=0\right\} .
$$

Note that the restriction of $\Phi=\Phi_{n}$ to $R$ is injective since the linear span of $R$ intersects $\mathbb{Z}(-3,0,0)$, see the definition of $\Phi$, in exactly one element, namely $(0,0,0)$. Therefore, it is enough to enumerate $|\Phi(R)|$. We claim that

$$
\Phi(R)=T_{n} \cup T_{n-3} \cup T_{n-6} \cup \ldots
$$


(note that this union is automatically disjoint), which is a reformulation of the assertion of the lemma due to the rule of sum.

We have $\Phi(R) \subset T_{n} \cup T_{n-1} \cup T_{n-2} \cup \ldots$ by Lemma 12 . As the first coordinate of each $v \in R$ is zero and $\Phi(v)-v \in \mathbb{Z}(-3,0,0)$ by definition, it follows that the first coordinate of $\Phi(v)$, for $v \in R$, must be divisible by 3 , that is $\Phi(R) \subset T_{n} \cup T_{n-3} \cup T_{n-6} \cup \ldots$.

For the inverse inclusion, take some $v=(n-2 a-3 b, a, b) \in T_{n-3 j}$, for some $j \geq 0$. Then, $n-2 a-3 b=3 j$, by definition. Clearly, $v \prec n \mathbf{e}(1)$. If $j=0$, then $v \in R$ and $\Phi(v)=v$. If $j>0$, then we apply to $v$ the following procedure $j$ times: first add $(-2,1,0)$, then add $(-1,-1,1)$, then add $(0,0,-1)$. It is easy to see that, by doing this, we follow the order $\prec$ inside $I(n \mathbf{e}(1))$. As $(-3,0,0)=(-2,1,0)+(-1,-1,1)+(0,0,-1)$, as the result, we obtain $(0, a, b) \prec v$. In particular, we have $(0, a, b) \in R$. From the definition of $\Phi$ and Lemma 12, we obtain $\Phi((0, a, b))=v$. Therefore $T_{n} \cup T_{n-3} \cup T_{n-6} \cup \cdots \subset \Phi(R)$. This proves (3.7) and completes the proof of the lemma.

To prove our theorem, we need to prove (3.4). Lemma 13 corresponds to the last two factors on the right-hand side of (3.4), formula (3.6) corresponds to the first factor and Lemma 14 corresponds to the second factor. The claim of the theorem now follows using the rule of sum. The intuitive picture behind this proof is given in Figure 4.

As a direct consequence of Theorem 11 and [3,4], we get:

Corollary 15. For $i \in \mathbb{N}$, we have:

$$
\begin{aligned}
C_{3(i-1)}^{(3)} & =\frac{1}{8}\left((i+1)\left(2 i^{2}+i+1\right)-\frac{1}{2}\left(1+(-1)^{i}\right)\right), \\
C_{3(i-1)+1}^{(3)} & =\frac{1}{8}\left((i+1)\left(2 i^{2}+3 i-1\right)+\frac{1}{2}\left(1+(-1)^{i}\right)\right), \\
C_{3(i-1)+2}^{(3)} & =\frac{1}{8}\left((i+1)\left(2 i^{2}+5 i+1\right)-\frac{1}{2}\left(1+(-1)^{i}\right)\right) .
\end{aligned}
$$

\section{4. $C_{n}^{(3)}$ and hollow hexagons}

\subsection{Triangular tilings}

Consider a regular triangular tiling of a Euclidean plane as shown in Figure 5. We assume that the side of the basic equilateral triangle (the fundamental region) of this tiling has length 1 . Each intersection point if called a vertex of the tiling. Each straight line of the tiling is called a tiling line. A horizontal tiling line will be called a line of type 1 . A tiling line of type 2 is a tiling line obtained from a tiling line of type 1 by a clockwise rotation by $\frac{\pi}{3}$. A tiling line of type 3 is a tiling line obtained from a tiling line of type 1 by a clockwise rotation by $\frac{2 \pi}{3}$.

\subsection{T-hexagons and their h-envelopes}

For $i=1,2,3$, a tiling strip of type $i$ is the region between two tiling lines of type $i$, including these lines (see Figure 5 for an example of a tiling strip of type 2). In particular, if these two lines coincide, then the corresponding tiling strip coincides with each of these tiling lines. A t-hexagon is, by definition, the 


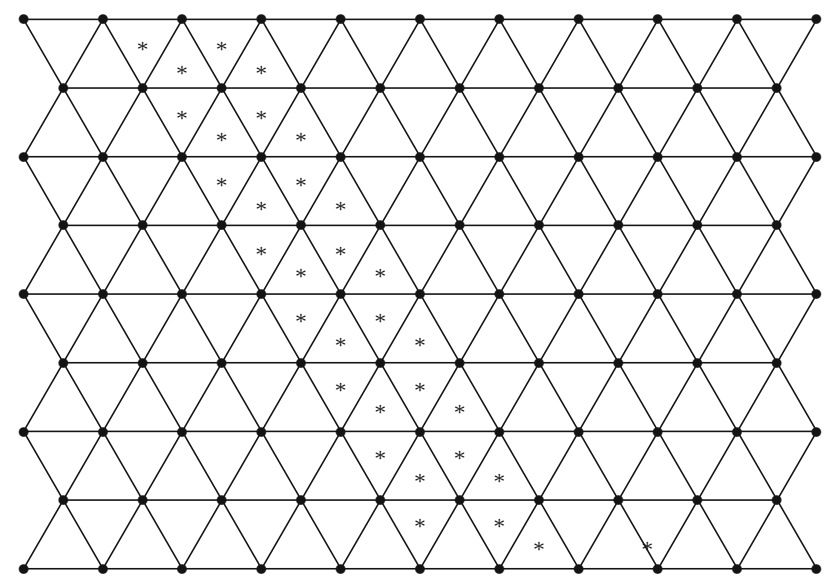

FiguRE 5. Triangular tiling, vertices, and tiling lines; triangles marked with $*$ form a tiling strip of type 2

intersection of three tiling strips, one for each type. Note that a t-hexagon can be:

- empty;

- equal to a vertex of the tiling;

- equal to a bounded line segment of a tiling line;

- a polygon with three, four, five, or six vertices.

By the perimeter of a t-hexagon we mean its perimeter as a polygon. Clearly, each t-hexagon has finite perimeter. The perimeter of a vertex is zero, while the perimeter of a bounded line segment is twice the length of this line segment.

The group of symmetries of the triangular tiling is the triangle group

$$
\Delta(3,3,3)=\left\langle a, b, c: a^{2}=b^{2}=c^{2}=(a b)^{3}=(b c)^{3}=(c a)^{3}=1\right\rangle
$$

generated by reflections with respect to the sides of the fundamental region of the tiling. Two t-hexagons which can be obtained from each other applying some element in $\Delta(3,3,3)$ will be called isomorphic. For $n \in \mathbb{Z}_{\geq 0}$, we denote by $T_{n}$ the number of isomorphism classes of thexagons with perimeter $2 n$.

Centroids of tiling triangles form a dual hexagonal tiling of our plane. Given a t-hexagon $H$, its hexagonal envelope $E(H)$ is the union of all hexagons in the hexagonal tiling which intersect $H$, see Figure 6 for an example of a t-hexagon (bold lines) and its hexagonal envelope (dotted lines).

Lemma 16. Let $H$ be a t-hexagon of perimeter $i$ for some $i \in \mathbb{Z}_{\geq 0}$. Then, the hexagonal envelope of $H$ has $6+2 i$ vertices.

Proof. For $i=0,1,2,3,4,5$ the statement of the lemma follows by inspecting all t-hexagons of perimeter $i$. These t-hexagons are given in the following list:

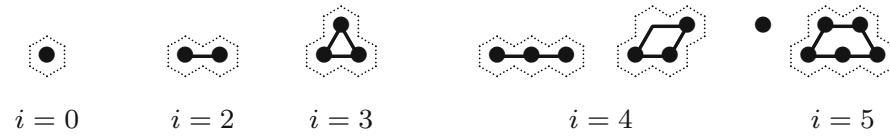




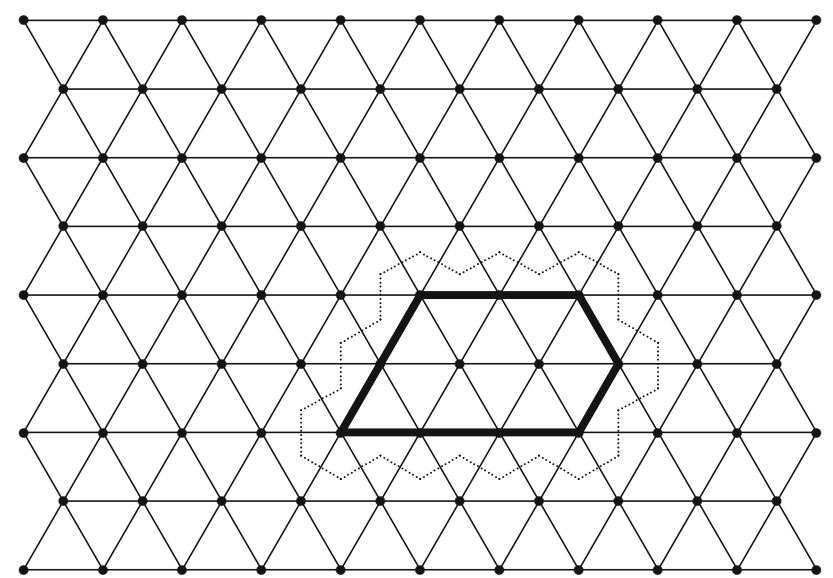

Figure 6. A t-hexagon and its hexagonal envelope

We claim that the rest follows by induction on $i$. Indeed, assume that $H$ is the intersection of three tiling strips (one for each type). We can, in turn, pull the lines defining these strips closer to each other, one step at a time. Eventually, by one such step, we will get a smaller t-hexagon $H^{\prime}$. There are four possible cases.

Case 1. The t-hexagon $H^{\prime}$ is obtained from $H$ by collapsing a line segment to a vertex as illustrated here:

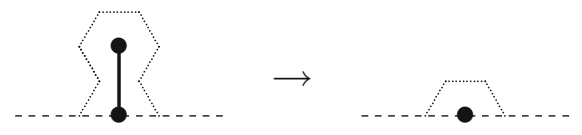

In this case, we see that the perimeter of $H$ decreases by 2 and the number of vertices of the hexagonal envelope decreases by 4 .

Case 2. The t-hexagon $H^{\prime}$ is obtained from $H$ by collapsing a trapezoid segment onto its basis as illustrated here (the length of the segment can be arbitrary):

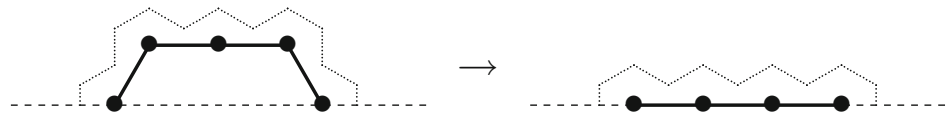

In this case, we see that the perimeter of $H$ decreases by 1 and the number of vertices of the hexagonal envelope by 2 .

Case 3. The t-hexagon $H^{\prime}$ is obtained from $H$ by collapsing a trapezoid segment onto its basis as illustrated here (the length of the segment can be arbitrary): 


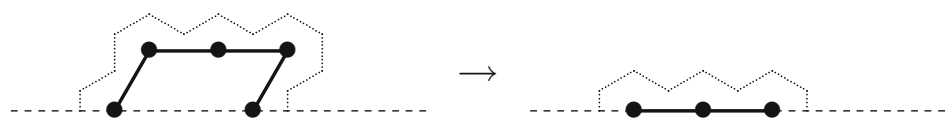

In this case, we see that the perimeter of $H$ decreases by 2 and the number of vertices of the hexagonal envelope by 4 .

Case 4. The t-hexagon $H^{\prime}$ is obtained from $H$ by collapsing a trapezoid segment to its basis as illustrated here:

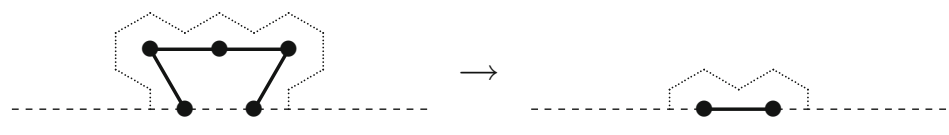

In this case, we see that the perimeter of $H$ decreases by 3 and the number of vertices of the hexagonal envelope by 6 .

Since all the above changes agree, by linearity, with the desired formula, the claim of the lemma follows by induction.

Hexagonal envelopes of t-hexagons seem to be exactly the hollow hexagons considered in $[3,4]$ (the latter papers do not really have any mathematically precise definition of hollow hexagons).

\subsection{Characters of t-hexagons}

We would like to encode t-hexagons using vectors with non-negative integral coordinates. For this, we will need some notation. Denote by $v_{1}, v_{2}$ and $v_{3}$, the vectors in the Euclidean plane as shown in Figure 7. Note that all these vectors have length one and that $v_{1}+v_{2}+v_{3}=0$. In Figure 7 , we also see a $*-$ marked t-hexagon which is the intersection of the tiling strips formed by thick lines. The tiling lines which bound the tiling strips are marked by numbers $1,2,3,4,5,6$ which correspond to going along the perimeter of the hexagon starting from the bottom side and going into the clockwise direction.

Let now $H$ be a nonempty t-hexagon given as the intersection of three tiling strips, one for each type. Without loss of generality, we may assume that each tiling line which bounds each of these tiling strips has a nonempty intersection with $H$. We number the tiling lines forming the boundaries of the tiling strips in the same way as in Figure 7. Note that, if two tiling lines coincide, we still count them as two different lines in our numbering. This corresponds to walking along the boundary of $H$, starting with the bottom side, first along $v_{1}$, then along $-v_{3}$, then along $v_{2}$, then along $-v_{1}$, then along $v_{3}$ and, finally, along $-v_{2}$.

The intersection of a boundary tiling line of a tiling strip with $H$ is then either a vertex or a side of $H$. We denote by $\chi(H)$ the vector $\left(a_{1}, a_{2}, a_{3}, a_{4}, a_{5}, a_{6}\right)$ where for $i=1,2,3,4,5,6$ the number $a_{i}$ is the length of the intersection of the line $i$ with $H$. For example, for the $*$-marked t-hexagon in Figure 7 , we have $\chi(H)=(1,1,1,1,1,1)$, while for the thick t-hexagon in Figure 6 , we have $\chi(H)=(3,0,2,2,1,1)$. The vector $\chi(H)$ will be called the character of $H$.

One could also give a description of $\chi(H)$ as follows: Start with the rightmost vertex on the bottom edge of $H$. Walk along $v_{1}$ until the next vertex 


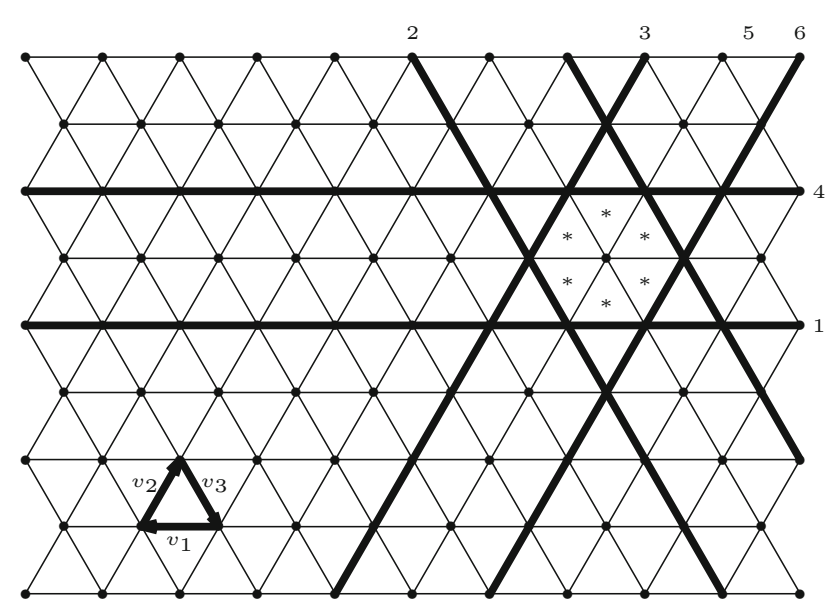

FiguRE 7. Basic vectors and lines

(which might coincide with the starting one). The number $a_{1}$ is the length of this walk. Continue along $-v_{3}$ to record $a_{2}$, then along $v_{2}$ to record $a_{3}$ and so on in the order described above.

The original action of $\Delta(3,3,3)$ induces an action on the set of characters of t-hexagons which is generated by the cyclic permutations of components of the character and the flip

$$
\left(a_{1}, a_{2}, a_{3}, a_{4}, a_{5}, a_{6}\right) \mapsto\left(a_{6}, a_{5}, a_{4}, a_{3}, a_{2}, a_{1}\right) .
$$

Using this action, we can change $H$ to an isomorphic t-hexagon $H^{\prime}$ such that we have $\chi\left(H^{\prime}\right)=\left(a_{1}, a_{2}, a_{3}, a_{4}, a_{5}, a_{6}\right)$ where the following conditions are satisfied:

$$
a_{1}+a_{3}+a_{5} \leq a_{2}+a_{4}+a_{6} \quad \text { and } \quad a_{1} \geq a_{3} \geq a_{5}
$$

Such $H^{\prime}$ as well as its character will be called distinguished. It is easy to see that a distinguished representative in the isomorphism class of $H$ is unique up to shift of tiling. As an example, the regular hexagon in Figure 7 is distinguished, while the t-hexagon in Figure 7 is not distinguished since the first inequality in (4.1) fails.

Note that our walk along the perimeter of $H$ always returns to the original point. From this, it follows that $\left(a_{1}, a_{2}, a_{3}, a_{4}, a_{5}, a_{6}\right) \in \mathbb{Z}_{\geq 0}^{6}$ is the character of some t-hexagon if and only if

$$
\left(a_{1}-a_{4}\right) v_{1}+\left(a_{5}-a_{2}\right) v_{3}+\left(a_{3}-a_{6}\right) v_{2}=0 .
$$

Taking into account $v_{3}=-v_{1}-v_{2}$ and linear independence of $v_{1}$ and $v_{2}$, Equation (4.2) is equivalent to

$$
a_{1}+a_{2}-a_{4}-a_{5}=0 \text { and } a_{2}+a_{3}-a_{5}-a_{6}=0 .
$$

Lemma 17. Let $H$ be a distinguished t-hexagon and $\chi(H)=\left(a_{1}, a_{2}, a_{3}, a_{4}, a_{5}, a_{6}\right)$. Then, we have

$$
a_{1} \leq a_{4}, \quad a_{5} \leq a_{2} \quad \text { and } \quad a_{3} \leq a_{6}
$$


Proof. From Equation (4.3), we have $a_{1}-a_{4}=a_{5}-a_{2}=a_{3}-a_{6}$. Plugging $a_{1}-a_{4}=a_{5}-a_{2}$ into the first inequality in (4.1) implies $a_{3} \leq a_{6}$. Plugging $a_{5}-a_{2}=a_{3}-a_{6}$ into the first inequality in (4.1) implies $a_{1} \leq a_{4}$. Plugging $a_{1}-a_{4}=a_{3}-a_{6}$ into the first inequality in (4.1) implies $a_{5} \leq a_{2}$.

\subsection{Elementary operations on distinguished t-hexagons}

Let $H$ be a distinguished t-hexagon and $\chi(H)=\left(a_{1}, a_{2}, a_{3}, a_{4}, a_{5}, a_{6}\right)$. We consider four elementary operations on hexagons.

Operation $\boldsymbol{\Phi}$. Assume $a_{1}-a_{3} \geq 2$. Then, using Lemma 17 , it is easy to check that the vector

$$
\left(a_{1}-1, a_{2}, a_{3}+1, a_{4}-1, a_{5}, a_{6}+1\right)
$$

satisfies all conditions in (4.1) and (4.3) and hence is the character of a unique distinguished t-hexagon which we denote by $\Phi(H)$. Here is an example of this operation:
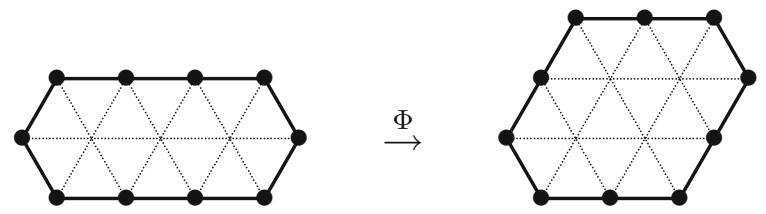

Operation $\boldsymbol{\Psi}$. Assume $a_{1}>a_{3}>a_{5}$. Then, using Lemma 17, it is easy to check that the vector

$$
\left(a_{1}-1, a_{2}+1, a_{3}, a_{4}-1, a_{5}+1, a_{6}\right)
$$

satisfies all conditions in (4.1) and (4.3) and hence is the character of a unique distinguished t-hexagon which we denote by $\Psi(H)$. Here is an example of this operation:
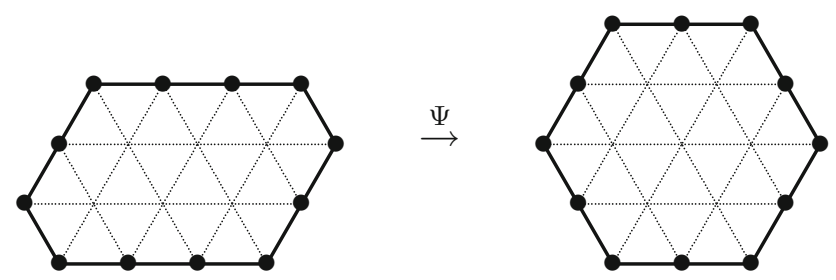

Operation $\Theta$. Assume $a_{5}>0$. Then, using Lemma 17, it is easy to check that the vector

$$
\left(a_{1}-1, a_{2}+1, a_{3}-1, a_{4}+1, a_{5}-1, a_{6}+1\right)
$$

satisfies all conditions in (4.1) and (4.3) and hence is the character of a unique distinguished t-hexagon which we denote by $\Theta(H)$. Here is an example of this operation: 

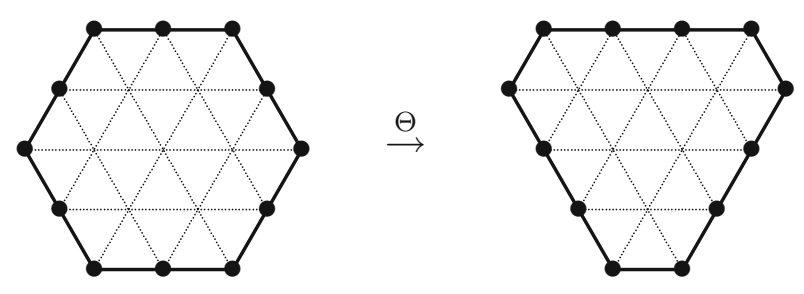

Operation $\Lambda$. Assume $a_{3}-a_{5} \geq 2$. Then, using Lemma 17, it is easy to check that the vector

$$
\left(a_{1}-1, a_{2}+2, a_{3}-2, a_{4}+1, a_{5}, a_{6}\right)
$$

satisfies all conditions in (4.1) and (4.3) and hence is the character of a unique distinguished t-hexagon which we denote by $\Lambda(H)$. Here is an example of this operation:
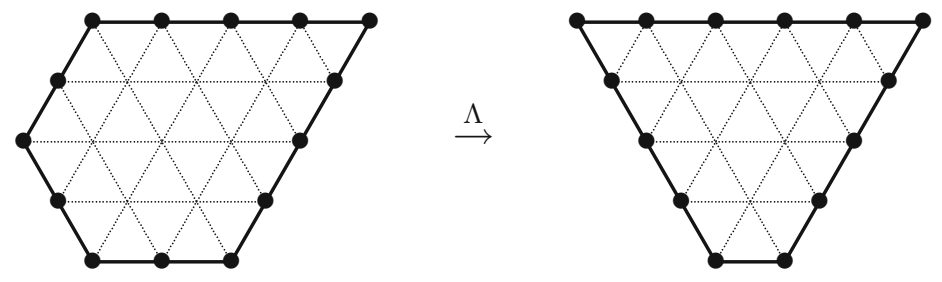

Directly from the definitions, it is easy to see that all maps $\Phi, \Psi, \Theta$ and $\Lambda$ do not change the perimeter. As illustrated by the examples, all these maps have rather transparent geometric interpretations which could be obtained by moving the boundary tiling lines of the tiling strips which define the original t-hexagon.

\subsection{Signature and defect}

Let $H$ be a distinguished t-hexagon. Assume that $\chi(H)=\left(a_{1}, a_{2}, a_{3}, a_{4}, a_{5}, a_{6}\right)$. Then, the vector $\operatorname{sign}(H):=\left(a_{1}-a_{3}, a_{3}-a_{5}, a_{5}\right) \in \mathbb{Z}_{\geq 0}^{3}$ will be called the signature of $H$. For example, the regular hexagon in Figure 7 has signature $(0,0,1)$ while a distinguished t-hexagon isomorphic to the t-hexagon in Figure 6 has signature $(1,1,0)$. Directly from the definitions, one computes that for any distinguished t-hexagon $H$, we have:

$$
\begin{aligned}
& \operatorname{sign}(\Phi(H))=\operatorname{sign}(H)+(-2,1,0), \\
& \operatorname{sign}(\Psi(H))=\operatorname{sign}(H)+(-1,-1,1), \\
& \operatorname{sign}(\Theta(H))=\operatorname{sign}(H)+(0,0,-1), \\
& \operatorname{sign}(\Lambda(H))=\operatorname{sign}(H)+(1,-2,0),
\end{aligned}
$$

provided that the thexagons $\Phi(H), \Psi(H), \Theta(H)$ or, respectively, $\Lambda(H)$, are defined.

We define the defect of $H$ as

$$
\operatorname{def}(H):=a_{2}+a_{4}+a_{6}-a_{1}-a_{3}-a_{5}
$$

and note that the defect of a distinguished t-hexagon is always non-negative. 


\subsection{The number of thexagons}

Our main result in this section is the following statement which gives a direct connection between the present paper and $[3,4]$.

Theorem 18. For all $n \in \mathbb{Z}_{\geq 0}$, mapping $H$ to $\operatorname{sign}(H)$ induces a bijection between the set of isomorphism classes of distinguished t-hexagons of perimeter $2 n$ and the set $I(n \mathbf{e}(1))$.

Proof. Consider a distinguished t-hexagon $Q$ having the character $(n, 0,0, n, 0,0)$. The signature of this t-hexagon is $(n, 0,0)=n \mathbf{e}(1)$. Applying, whenever possible, a sequence of operations $\Phi, \Psi, \Theta$ and $\Lambda$ to $Q$, produces a set of thexagons of perimeter $2 n$. From (4.4), it follows that the set of signatures for all thexagons which can be obtained in this way is contained in $I(n \mathbf{e}(1))$. Observe that the operation $\Phi$ is defined as soon as $a_{1}-a_{3} \geq 2$ and that this condition is equivalent to the fact that adding $(-2,1,0)$ to the signature of the input t-hexagon gives a vector with non-negative coordinates. Put differently, if we have a t-hexagon such that the sum of its signature and $(-2,1,0)$ has non-negative coordinates, then $\Phi$ can be applied to this t-hexagon. Similar observations also apply to $\Psi$ and $(-1,-1,1)$, to $\Theta$ and $(0,0,-1)$ and to $\Lambda$ and $(1,-2,0)$. Consequently, the set of signatures for all t-hexagons which can be obtained from $Q$ by all possible sequences of $\Phi, \Psi, \Theta$ and $\Lambda$ coincides with $I(n \mathbf{e}(1))$.

Our next step is to show that each distinguished t-hexagon of perimeter $2 n$ can be obtained from $Q$ using a sequence of operations of the form $\Phi, \Psi$, $\Theta$ and $\Lambda$ (in fact, the first three would suffice). Let $K$ be a distinguished thexagon of perimeter $2 n$ with character $\left(a_{1}, a_{2}, a_{3}, a_{4}, a_{5}, a_{6}\right)$. Assume $a_{5}>0$. Then, using Lemma 17, it is easy to check that the vector

$$
\left(a_{1}+1, a_{2}-1, a_{3}, a_{4}+1, a_{5}-1, a_{6}\right)
$$

satisfies all conditions in (4.1) and (4.3) and hence is the character of a unique distinguished t-hexagon. Therefore, $K=\Psi\left(K^{\prime}\right)$ for some distinguished thexagon $K^{\prime}$ and the character of $K^{\prime}$ has a smaller fifth coordinate. In particular, $K$ is obtained, using a sequence of $\Psi$ 's, from some distinguished t-hexagon $K^{\prime}$, the character of which has zero-fifth coordinate.

Let $K$ be a distinguished t-hexagon of perimeter $2 n$ with character $\left(a_{1}, a_{2}, a_{3}, a_{4}, a_{5}, a_{6}\right)$. Assume that $a_{5}=0$ and $a_{3}>0$. Then, using Lemma 17 , it is easy to check that the vector

$$
\left(a_{1}+1, a_{2}, a_{3}-1, a_{4}+1, a_{5}, a_{6}-1\right)
$$

satisfies all conditions in (4.1) and (4.3) and hence is the character of a unique distinguished t-hexagon. Therefore, $K=\Phi\left(K^{\prime}\right)$ for some distinguished thexagon $K^{\prime}$ and the character of $K^{\prime}$ has a smaller third coordinate. In particular, $K$ is obtained, using a sequence of $\Phi$ 's and $\Psi$ 's, from some distinguished t-hexagon $K^{\prime}$ the character of which has zero-third and fifth coordinates.

Let $K$ be a distinguished t-hexagon of perimeter $2 n$ with character $\left(a_{1}, a_{2}, 0, a_{4}, 0, a_{6}\right)$. Then, $a_{4} \geq a_{1}$ by Lemma 17. If $a_{4}=a_{1}$, then from (4.3) it follows that $a_{2}=a_{6}=0$ and $K=Q$. If $a_{4}>a_{1}$, then from (4.3) it follows 
that $a_{2}=a_{6}=a_{4}-a_{1}>0$. If $a_{4}>a_{1}+1$, then, using Lemma 17, it is easy to check that the vector

$$
\left(b_{1}, b_{2}, b_{3}, b_{4}, b_{5}, b_{6}\right):=\left(a_{1}+1, a_{2}-1, a_{3}+1, a_{4}-1, a_{5}+1, a_{6}-1\right)
$$

satisfies all conditions in (4.1) and (4.3) and hence is the character of a unique distinguished t-hexagon, say $K^{\prime}$. Note that, by construction, $\operatorname{def}\left(K^{\prime}\right)<\operatorname{def}(K)$ and that $K=\Theta\left(K^{\prime}\right)$. Finally, if $a_{4}=a_{1}+1$, then from (4.3) it follows that the character of $K$ is of the form $(x, 1,0, x+1,0,1)$, for some $x$. In this case, the perimeter of $K$ is $2 x+3$, which is an odd number, contradicting our assumptions. Therefore, the case $a_{4}=a_{1}+1$ cannot occur.

Using induction on defect and the above steps, it follows that any distinguished t-hexagon of perimeter $2 n$ is obtained using $\Phi, \Psi$ and $\Theta$ from a distinguished t-hexagon of perimeter $2 n$ with character $\left(a_{1}, 0,0, a_{4}, 0,0\right)$. But from Equation 4.3, it thus follows that $a_{1}=a_{4}=n$, and hence, the latter t-hexagon must be isomorphic to $Q$.

As a consequence of the above argument, we have that the image of the signature map is contained in $I(n \mathbf{e}(1))$. So, it remains to show that the signature map is injective.

Let $K$ be a distinguished t-hexagon with signature $(x, y, z)$ and of perimeter $2 n$. Then, the character of $K$ equals $\left(x+y+z, a_{2}, y+z, a_{4}, z, a_{6}\right)$ for some $a_{2}, a_{4}, a_{6} \in \mathbb{Z}_{\geq 0}$. From (4.3), we have

$$
x+y+z-a_{4}=z-a_{2}=y+z-a_{6} .
$$

Since the perimeter of $K$ is $2 n$, we also have

$$
x+y+z+a_{2}+y+z+a_{4}+z+a_{6}=2 n
$$

and hence $a_{2}, a_{4}$ and $a_{6}$ are uniquely determined. This means that the character of $K$ is uniquely determined and thus $K$ is uniquely determined up to isomorphism by its signature. This completes the proof.

As an immediate corollary from Theorem 18, we have:

Corollary 19. For all $n \in \mathbb{Z}_{\geq 0}, C_{n}^{(3)}$ is the number of isomorphism classes of t-hexagons with perimeter $2 n$.

Our proof of Theorem 18 provides another connection to the sequence $A 001399(n)$ giving the number of partitions of $n$ in at most three parts which was already mentioned in Subsection 3.4. Let $P_{n}$ denote the set of all partitions of $n$ in at most three parts. If $n<0$, we set $P_{n}=\varnothing$.

Corollary 20. Let $n \in \mathbb{Z}_{\geq 0}$. Mapping $H$ with $\chi(H)=\left(a_{1}, a_{2}, a_{3}, a_{4}, a_{5}, a_{6}\right)$ to $\left(a_{1}, a_{3}, a_{5}\right)$ induces a bijection between the set of isomorphism classes of distinguished t-hexagons of perimeter $2 n$ and the set $P_{n} \cup P_{n-3} \cup P_{n-6} \cup \ldots$. In particular, we have

$$
C_{n}^{(3)}=A 001399(n)+A 001399(n-3)+A 001399(n-6)+\ldots
$$

Proof. Restricting the bijection constructed in the proof of Theorem 18 to the set of distinguished t-hexagons of defect $2 i$ and thereafter mapping $\operatorname{sign}(H)=$ $(x, y, z)$ to the partition $(x+y+z, y+z, z)$ of $x+2 y+3 z$, provides a bijection from the set of distinguished t-hexagons of defect $2 i$ to $P_{n-i}$. 


\section{Partitions modulo $d$}

\subsection{Partitions and refinement}

For $n \in \mathbb{Z}_{\geq 0}$ denote by $\Pi_{n}$ the set of all partitions of $n$, that is, the set of all tuples $\boldsymbol{\lambda}=\left(\lambda_{1}, \lambda_{2}, \ldots, \lambda_{k}\right)$ such that $\lambda_{1}, \lambda_{2}, \ldots, \lambda_{k} \in \mathbb{N}, n=\lambda_{1}+\lambda_{2}+\cdots+\lambda_{k}$ and $\lambda_{1} \geq \lambda_{2} \geq \cdots \geq \lambda_{k}$. As usual, we write $\boldsymbol{\lambda} \vdash n$ for $\boldsymbol{\lambda} \in \Pi_{n}$.

For $\boldsymbol{\lambda}=\left(\lambda_{1}, \lambda_{2}, \ldots, \lambda_{k}\right) \vdash n$ and $\boldsymbol{\mu}=\left(\mu_{1}, \mu_{2}, \ldots, \mu_{l}\right) \vdash n$, we say that $\boldsymbol{\lambda}$ refines $\boldsymbol{\mu}$ and write $\boldsymbol{\mu}<\boldsymbol{\lambda}$ provided that $l<k$ and there is a partition $J_{1} \cup J_{2} \cup \cdots \cup J_{l}$ of $\{1,2, \ldots, k\}$ into a disjoint union of non-empty subsets such that

$$
\mu_{i}=\sum_{j \in J_{i}} \lambda_{j}, \quad \text { for all } i=1,2, \ldots, l .
$$

The partially ordered set $\left(\Pi_{n},<\right)$ was studied in $[1,2,23]$. In particular, in [23], it was shown that it has some nasty properties. We refer the reader to [23] for more details on this poset.

The poset $\left(\Pi_{n},<\right)$ is graded with respect to the rank function $\left(\lambda_{1}, \lambda_{2}, \ldots, \lambda_{k}\right) \mapsto k$.

\subsection{Partitions modulo $d$}

For $d \in \mathbb{N}$, define an equivalence relation $\sim_{d}$ on $\Pi_{n}$ as follows: Given $\boldsymbol{\lambda}=$ $\left(\lambda_{1}, \lambda_{2}, \ldots, \lambda_{k}\right) \vdash n$ and $\boldsymbol{\mu}=\left(\mu_{1}, \mu_{2}, \ldots, \mu_{l}\right) \vdash n$ set $\boldsymbol{\lambda} \sim_{d} \boldsymbol{\mu}$ provided that $k=l$ and there is $\pi \in S_{k}$ such that $d$ divides $\lambda_{i}-\mu_{\pi(i)}$, for all $i$. In other words, $\boldsymbol{\lambda} \sim_{d} \boldsymbol{\mu}$ if and only if the multisets of residues modulo $d$ for parts of $\boldsymbol{\lambda}$ and $\boldsymbol{\mu}$ coincide. For $\boldsymbol{\lambda} \vdash n$, we denote the $\sim_{d^{-}}$class of $\boldsymbol{\lambda}$ by $\overline{\boldsymbol{\lambda}}^{(d)}$.

Since $\sim_{d}$-equivalent partitions have the same number of parts and partitions with the same number of parts are incomparable with respect to the refinement order $<$, this order induces a partial order $<_{d}$ on the set $\Pi_{n, d}:=$ $\Pi_{n} / \sim_{d}$ defined as the transitive closure of the relation $\tilde{<}$ given by $\overline{\boldsymbol{\lambda}} \tilde{<} \overline{\boldsymbol{\mu}}$ if there are $\boldsymbol{\lambda}^{\prime} \in \overline{\boldsymbol{\lambda}}$ and $\boldsymbol{\mu}^{\prime} \in \overline{\boldsymbol{\mu}}$ such that $\boldsymbol{\lambda}^{\prime}<\boldsymbol{\mu}^{\prime}$. The poset $\Pi_{n, d}$ inherits from $\Pi_{n}$ the structure of a graded poset.

Define the poset $\Pi_{n, d}^{*}$ as follows: if $d$ does not divide $n$, set $\Pi_{n, d}^{*}:=$ $\Pi_{n, d}$ with the order $<_{d}$; if $d$ divides $n$, define $\Pi_{n, d}^{*}$ as the poset obtained from $\left(\Pi_{n, d},<_{d}\right)$ by adding a minimum element, denoted $\varnothing$ (for simplicity, we will keep the notation $<_{d}$ for the partial order on $\left.\Pi_{n, d}^{*}\right)$. The structure of a graded poset on $\Pi_{n}$ induces the structure of a graded poset on $\Pi_{n, d}^{*}$ by defining the degree of $\varnothing$ to be zero. The class $\overline{(1,1, \ldots, 1)}^{(d)}=\{(1,1, \ldots, 1)\}$ of the partition $(1,1, \ldots, 1)$ is the maximum element in $\Pi_{n, d}^{*}$.

\section{3. $\Pi_{n, d}^{*}$ versus $\mathscr{P}_{d}$}

Our main result in this section is the following:

Theorem 21. The (graded) posets $\left(\Pi_{n, d}^{*},<_{d}\right)$ and $(I(n \mathbf{e}(1)), \prec)$ are isomorphic.

Proof. To each $\boldsymbol{\lambda} \vdash n$, we associate the vector $\left(v_{1}^{\boldsymbol{\lambda}}, v_{2}^{\boldsymbol{\lambda}}, \ldots, v_{d}^{\boldsymbol{\lambda}}\right)$, where, for $i=1,2, \ldots, d$, we have

$$
v_{i}^{\lambda}:=\left|\left\{j: \lambda_{j} \equiv i \bmod d\right\}\right| .
$$


This map is constant on the $\sim_{d}$-equivalence classes and hence induces a map from $\Pi_{n, d}$ to $\mathscr{P}_{d}$. We extend this map to $\Pi_{n, d}^{*}$ by sending the $\varnothing$ element to the zero vector in case $d$ divides $n$. Denote the resulting map by $\Phi$. Note that $\Phi$ preserves the degree of an element, namely, it maps a partition with $k$ parts to a vector of height $k$.

First of all, we claim that $\Phi$ is a homomorphism of posets. Indeed, any refinement of partitions can be written as a composition of elementary refinements which simply refine one part of a smaller partition into two parts of a bigger partition. Such elementary refinement corresponds to the covering relation $\boldsymbol{\mu} \lessdot \boldsymbol{\lambda}$ where $\boldsymbol{\lambda}$ has $k$ parts while $\boldsymbol{\mu}$ has $k-1$ parts. Assume that this refines the part $\mu_{i}$ into parts $\lambda_{s}$ and $\lambda_{t}$. This means that $\mu_{i}=\lambda_{s}+\lambda_{t}$ and hence

$$
\mu_{i} \equiv \lambda_{s}+\lambda_{t} \bmod d
$$

Let $a, b, c \in\{1,2, \ldots, d\}$ be such that $\mu_{i} \equiv a, \lambda_{s} \equiv b$ and $\lambda_{t} \equiv c \bmod d$. Then, the element $\mathbf{e}(a)-\mathbf{e}(b)-\mathbf{e}(c)$ belong to $X_{d}$. This implies that $\Phi(\overline{\boldsymbol{\mu}}) \prec \Phi(\overline{\boldsymbol{\lambda}})$. It follows that $\Phi$ is a homomorphism of posets.

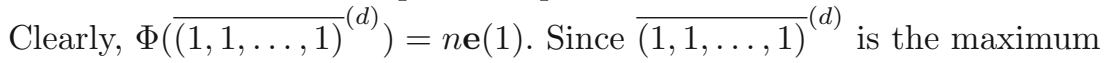
element in $\Pi_{n, d}^{*}$, it follows that $\Phi$ maps $\Pi_{n, d}^{*}$ to $I(n \mathbf{e}(1))$.

That $\Phi: \Pi_{n, d}^{*} \rightarrow I(n \mathbf{e}(1))$ is injective follows directly from the definition. It remains to show that $\Phi$ is surjective, in particular, invertible, and that $\Phi^{-1}$ is order preserving. We prove this by downward induction on the height $h$. If $h=d$, the claim is clear as $n \mathbf{e}(1)$ is the only element of $I(n \mathbf{e}(1))$ of height $h$.

For the induction step $h \rightarrow h-1$ let $\mathbf{v}$ and $\mathbf{w}$ be two elements in $I(n \mathbf{e}(1))$ of heights $h-1$ and $h$, respectively, and assume $\mathbf{v} \prec \mathbf{w}$. Then $\mathbf{v}=\mathbf{w}+\mathbf{x}$, for some $\mathbf{x} \in X_{d}$. Let $\mathbf{x}=\mathbf{e}(k)-\mathbf{e}(i)-\mathbf{e}(j)$, for some $i, j, k \in\{1,2, \ldots, d\}$. From the inductive assumption, there is $\boldsymbol{\lambda} \vdash n$ such that $\Phi(\overline{\boldsymbol{\lambda}})=\mathbf{w}$. Let $\lambda_{s}$ and $\lambda_{t}$ be two different parts of $\lambda$ with residues $i$ and $j$ modulo $d$, respectively. Define $\mu$ as the partition obtained from $\lambda$ by uniting $\lambda_{s}$ and $\lambda_{t}$. Then, $\Phi(\overline{\boldsymbol{\mu}})=\mathbf{v}$. Therefore, $\Phi$ is a bijection.

From the arguments above, it follows that the covering relations in $\Pi_{n, d}^{*}$ to $I(n \mathbf{e}(1))$ match precisely under $\Phi$. This implies that $\Phi$ is an isomorphism of posets, completing the proof of the theorem.

As an immediate corollary, we have:

Corollary 22. For $n \in \mathbb{Z}_{\geq 0}$ and $d \in \mathbb{N}$, we have $\left|\Pi_{n, d}^{*}\right|=C_{n}^{(d)}$.

\section{Connection to $d$-tonal partition monoid}

\subsection{Partition monoids}

For $n \in \mathbb{Z}_{>0}$, consider the sets $\underline{n}=\{1,2, \ldots, n\}$ and $\underline{n}^{\prime}=\left\{1^{\prime}, 2^{\prime}, \ldots, n^{\prime}\right\}$ (these two sets are automatically disjoint). Set $\mathbf{n}:=\underline{n} \cup \underline{n}^{\prime}$ and consider the $\operatorname{set} \mathcal{P}(\mathbf{n})$ of all partitions of $\mathbf{n}$ into a disjoint union of nonempty subsets. The cardinality of $\mathcal{P}(\mathbf{n})$ is the $2 n$-th Bell number, see $A 000110$ in [19]. 

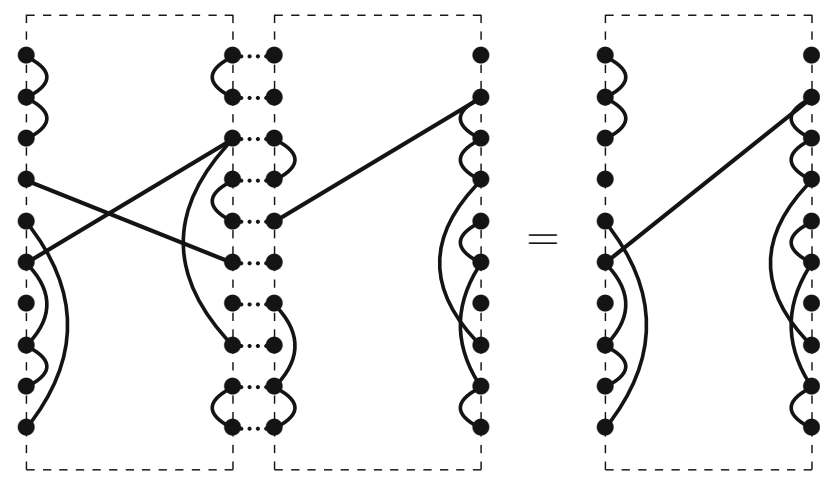

Figure 8. Partitions and their composition

The set $\mathcal{P}(\mathbf{n})$ has the natural structure of a monoid, see $[8,13,15,16]$. The composition $\sigma \circ \pi$ of two partitions $\sigma, \pi \in \mathcal{P}(\mathbf{n})$ is defined as follows (here $\underline{n}^{\prime \prime}=\left\{1^{\prime \prime}, 2^{\prime \prime}, \ldots, n^{\prime \prime}\right\}$ is disjoint from $\left.\mathbf{n}\right)$ :

- First consider the partition $\sigma^{\prime}$ of $\underline{n}^{\prime} \cup \underline{n}^{\prime \prime}$ which is induced from $\sigma$ via the bijection $\underline{n} \cup \underline{n}^{\prime} \rightarrow \underline{n}^{\prime} \cup \underline{n}^{\prime \prime}$ which sends $i \mapsto i^{\prime}$ for $i \in \underline{n}$ and $j^{\prime} \rightarrow j^{\prime \prime}$ for $j^{\prime} \in \underline{n}^{\prime}$.

- Let $\tilde{\pi}$ be the equivalence relation on $\underline{n} \cup \underline{n}^{\prime} \cup \underline{n}^{\prime \prime}$ whose parts are those of $\pi$ combined with singletons of $\underline{n}^{\prime \prime}$.

- Let $\tilde{\sigma}$ be the equivalence relation on $\underline{n} \cup \underline{n}^{\prime} \cup \underline{n}^{\prime \prime}$ whose parts are those of $\sigma^{\prime}$ combined with singletons of $\underline{n}$.

- Let $\tilde{\tau}$ denote the minimal (with respect to inclusions) equivalence relation on the set $\underline{n} \cup \underline{n}^{\prime} \cup \underline{n}^{\prime \prime}$ which contains both $\tilde{\pi}$ and $\tilde{\sigma}$.

- Let $\tilde{\tau}^{\prime}$ be the restriction of $\tilde{\tau}$ to $\underline{n} \cup \underline{n}^{\prime \prime}$.

- Define $\tau=\sigma \circ \pi$ as the partition of $\underline{n} \cup \underline{n}^{\prime}$ induced from the partition $\tilde{\tau}^{\prime}$ by the bijection $\underline{n} \cup \underline{n}^{\prime \prime} \rightarrow \underline{n} \cup \underline{n}^{\prime}$ which sends $i \mapsto i$ for $i \in \underline{n}$ and $j^{\prime \prime} \rightarrow j^{\prime}$ for $j^{\prime \prime} \in \underline{n}^{\prime \prime}$.

The identity element in the monoid $(\mathcal{P}(\mathbf{n}), \circ)$ is the identity partition

$$
\left\{\left\{1,1^{\prime}\right\},\left\{2,2^{\prime}\right\}, \ldots,\left\{k, k^{\prime}\right\}\right\} \in \mathcal{P}(\mathbf{k}) .
$$

Both elements of $\mathcal{P}(\mathbf{n})$ and the composition o admit a diagrammatic description as shown in Figure 8 (in the composition $\sigma \circ \pi$ which is the left-hand side of the equality in Figure 8, the element $\sigma$ is depicted on the left and the element $\pi$ on the right). We refer the reader to $[8,13,16]$ for further details.

\section{2. $d$-tonal partition monoids}

For $d \in \mathbb{N}$, the $d$-tonal partition monoid $\mathcal{P}_{d}(\mathbf{n})$, as introduced in [22], is the submonoid of $\mathcal{P}(\mathbf{n})$ which consists of all partitions $\sigma$ of $\mathbf{n}$ such that every part $\sigma_{i}$ of $\sigma$ satisfies the condition that

$$
d \quad \text { divides } \quad\left|\sigma_{i} \cap \underline{n}\right|-\left|\sigma_{i} \cap \underline{n}^{\prime}\right| .
$$


Thus, for $d=1$, we have $\mathcal{P}_{1}(\mathbf{n})=\mathcal{P}(\mathbf{n})$. For $d=2$, the above condition is equivalent to the requirement that all parts of $\sigma$ have even cardinality. Therefore, $\left|\mathcal{P}_{2}(\mathbf{n})\right|$ is given by the sequence $A 005046$ in [19] (see also [18]).

The twisted monoid algebra of the $d$-tonal partition monoid was studied (under various names) in [17,22], see also [9-11] for related algebras and [7, Section 5.1] for some recent developments. We record the following open problem:

Problem 23. Compute $\left|\mathcal{P}_{d}(\mathbf{n})\right|$ in a closed form as a function of $d$ and $n$.

As the twisted semigroup algebra of $\mathcal{P}_{d}(\mathbf{n})$ is generically semi-simple, see [22], and forms, for all $n$, a sequence of embedded algebras with multiplicityfree restrictions, see [9], there is a natural analogue of the Robinson-Schensted correspondence for $\mathcal{P}_{d}(\mathbf{n})$ and hence Problem 23 admits a combinatorial reformulation in terms of walks on a certain Bratelli diagram.

\subsection{Rank and $d$-signature}

For $\sigma \in \mathcal{P}_{d}(\mathbf{n})$, the $\operatorname{rank} \operatorname{rank}(\sigma)$ is the number of parts $\sigma_{i}$ in $\sigma$ such that both $\left|\sigma_{i} \cap \underline{n}\right| \neq 0$ and $\left|\sigma_{i} \cap \underline{n}^{\prime}\right| \neq 0$. Such parts are called propagating.

Note that, for $\sigma \in \mathcal{P}_{d}(\mathbf{n})$, the cardinality of any part of $\sigma$ which is entirely contained in $\underline{n}$ or in $\underline{n}^{\prime}$ is divisible by $d$.

Define the function $\Psi: \mathcal{P}_{d}(\mathbf{n}) \rightarrow \mathbb{Z}_{\geq 0}^{d}$, called the $d$-signature function, as follows: for $\sigma \in \mathcal{P}_{d}(\mathbf{n})$ define $\Psi(\sigma)=\left(v_{1}, v_{2}, \ldots, v_{d}\right)$, where for $i=1,2, \ldots, d$ the number $v_{i}$ is the number of parts $\sigma_{j}$ in $\sigma$ satisfying the conditions

$$
\left|\sigma_{j} \cap \underline{n}\right| \neq 0, \quad\left|\sigma_{j} \cap \underline{n}^{\prime}\right| \neq 0, \quad d \text { divides }\left|\sigma_{j} \cap \underline{n}\right|-i .
$$

Note that $v_{1}+v_{2}+\cdots+v_{d}=\operatorname{rank}(\sigma)$.

\section{4. $\mathcal{J}$-classes of $\boldsymbol{d}$-tonal partition monoids}

Two elements $\sigma, \pi \in \mathcal{P}_{d}(\mathbf{n})$ are called $\mathcal{J}$-equivalent, written $\sigma \mathcal{J} \pi$, provided that $\mathcal{P}_{d}(\mathbf{n}) \sigma \mathcal{P}_{d}(\mathbf{n})=\mathcal{P}_{d}(\mathbf{n}) \pi \mathcal{P}_{d}(\mathbf{n})$, see [5, Section 4.4]. For $\sigma \in \mathcal{P}_{d}(\mathbf{n})$ we denote by $\bar{\sigma}^{\mathcal{J}}$ the $\mathcal{J}$-equivalence class containing $\sigma$.

There is a natural partial order on the set $\mathcal{P}_{d}(\mathbf{n}) / \mathcal{J}$ given by inclusions: we write $\bar{\sigma}^{\mathcal{J}} \rightsquigarrow \bar{\pi}^{\mathcal{J}}$ if and only if $\mathcal{P}_{d}(\mathbf{n}) \sigma \mathcal{P}_{d}(\mathbf{n}) \subset \mathcal{P}_{d}(\mathbf{n}) \pi \mathcal{P}_{d}(\mathbf{n})$.

\subsection{Canonical elements}

An element $\sigma \in \mathcal{P}_{d}(\mathbf{n})$ will be called canonical provided that the following conditions are satisfied:

- Each part $\sigma_{i}$ of $\sigma$ satisfies $\left|\sigma_{i} \cap \underline{n}\right| \leq d$ and $\left|\sigma_{i} \cap \underline{n}^{\prime}\right| \leq d$.

- The intersections $\sigma_{i} \cap \underline{n}$ and $\sigma_{i} \cap \underline{n}^{\prime}$ are connected segments of $\underline{n}$ and $\underline{n}^{\prime}$, respectively, ordered by cardinalities of the intersections for those parts $\sigma_{i}$ which intersects both $\underline{n}$ and $\underline{n}^{\prime}$ and then followed by those parts of $\sigma$ which intersect only $\underline{n}$ or $\underline{n}^{\prime}$.

For example, the identity element in $\mathcal{P}_{d}(\mathbf{n})$ is canonical.

Lemma 24. For each $\sigma \in \mathcal{P}_{d}(\mathbf{n})$, there is a canonical $\pi \in \mathcal{P}_{d}(\mathbf{n})$ such that $\sigma \mathcal{J} \pi$. 

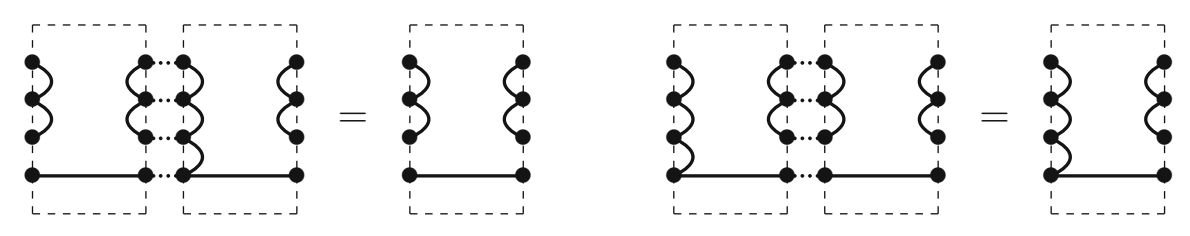

FiguRE 9. Illustration of the proof of Lemma 24

Proof. If some part $\sigma_{i}$ of $\sigma$ satisfies $\left|\sigma_{i} \cap \underline{n}\right|>d$ or $\left|\sigma_{i} \cap \underline{n}^{\prime}\right|>d$, then there is $\sigma^{\prime} \in$ $\mathcal{P}_{d}(\mathbf{n})$ which has exactly the same parts as $\sigma$ except for $\sigma_{i}$ which is split into two parts: a part with $d$ elements which is a subset of $\underline{n}$ (respectively, $\underline{n}^{\prime}$ ) and its complement. Existence of $\sigma^{\prime}$ follows using the construction shown in Figure 9 (in the case $d=3$ ) and note that $\sigma \mathcal{J} \sigma^{\prime}$ also follows from Figure 9. Proceeding inductively, we find an element $\tau \in \mathcal{P}_{d}(\mathbf{n})$ which is in the same $\mathcal{J}$-class as $\sigma$ and which satisfies the condition that $\left|\tau_{i} \cap \underline{n}\right| \leq d$ and $\left|\tau_{i} \cap \underline{n}^{\prime}\right| \leq d$ for each part $\tau_{i}$ of $\tau$. Permuting, if necessary, the elements of $\underline{n}$ and, independently, of $\underline{n}^{\prime}$ (that is, multiplying from the left and/or from the right by permutations, noting that all permutations belong to $\mathcal{P}_{d}(\mathbf{n})$ ), one rearranges $\tau$ into a canonical element $\pi$ in the same $\mathcal{J}$-class as $\sigma$. The claim follows.

Proposition 25. We have $\Psi\left(\mathcal{P}_{d}(\mathbf{n})\right)=I(n \mathbf{e}(1))$.

Proof. We use downward induction to prove that for each $k=n, n-1, n-$ $2, \ldots, 0$, the map $\Psi$ induces a bijection between the set of all canonical elements of rank $k$ in $\mathcal{P}_{d}(\mathbf{n})$ and the set of all elements of height $k$ in $I(n \mathbf{e}(1))$. The statement of the corollary then will follow from Lemma 24.

The basis of the induction is $k=n$. In this case, on the left-hand side, we have only one canonical element, the identity element, while on the right-hand side, we have $n \mathbf{e}(1)$ which is the image of the identity element under $\Psi$.

Let $\mathbf{v} \in I(n \mathbf{e}(1))$ be an element of height $k$ and let $\sigma$ be a canonical element such that $\Psi(\sigma)=\mathbf{v}$. Let $\mathbf{e}(k)-\mathbf{e}(i)-\mathbf{e}(j) \in X_{d}$ be such that $\mathbf{v}+$ $(\mathbf{e}(k)-\mathbf{e}(i)-\mathbf{e}(j)) \in I(n \mathbf{e}(1))$. Then, $\sigma$ has a part $\sigma_{s}$ such that $d$ divides $\left|\sigma_{s} \cap \underline{n}\right|-i$ and a different part $\sigma_{t}$ such that $d$ divides $\left|\sigma_{t} \cap \underline{n}\right|-j$. Consider the element $\sigma^{\prime}$ obtained from $\sigma$ by uniting $\sigma_{s}$ with $\sigma_{t}$ and keeping all other parts. Then, $\sigma^{\prime} \sigma=\sigma^{\prime}$ and hence $\mathcal{P}_{d}(\mathbf{n}) \sigma^{\prime} \mathcal{P}_{d}(\mathbf{n}) \subset \mathcal{P}_{d}(\mathbf{n}) \sigma \mathcal{P}_{d}(\mathbf{n})$. Moreover, $\Psi\left(\sigma^{\prime}\right)=\mathbf{v}+\mathbf{e}(k)-\mathbf{e}(i)-\mathbf{e}(j)$. This implies surjectivity of the induction step.

At the same time, the form of the canonical element immediately implies that it is obtained from the identity element using the unification procedure described in the previous paragraph, followed by splitting off $d$-element parts contained in $\underline{n}$ or $\underline{n}^{\prime}$ (note that the latter parts do not affect the value of $\Psi$ by definition). This implies that $\Psi$ takes values inside $I(n \mathbf{e}(1))$ and completes the proof.

Corollary 26. For each $\sigma \in \mathcal{P}_{d}(\mathbf{n})$, there is a unique canonical $\pi \in \mathcal{P}_{d}(\mathbf{n})$ such that $\sigma \mathcal{J} \pi$. 
Proof. Taking into account Proposition 25, the claim follows from the observation that different canonical elements are sent by $\Psi$ to different elements in $I(n \mathbf{e}(1))$.

\subsection{A combinatorial description of the $\mathcal{J}$-order}

Our second main result, which explains our interest in $C_{n}^{(d)}$, is the following:

Theorem 27. The map $\Psi:\left(\mathcal{P}_{d}(\mathbf{n}) / \mathcal{J}, \rightsquigarrow\right) \rightarrow(I(n \mathbf{e}(1)), \prec)$ is an isomorphism of posets.

Proof. From Proposition 25, we have a map $\Psi: \mathcal{P}_{d}(\mathbf{n}) / \mathcal{J} \rightarrow I(n \mathbf{e}(1))$. This map is bijective by the combination of Proposition 25 and Corollary 26. From the third paragraph of the proof of Proposition 25, it follows that for each pair of elements $\mathbf{v}, \mathbf{w} \in I(n \mathbf{e}(1))$ such that $\mathbf{v} \prec \mathbf{w}$, there are $\sigma, \pi \in \mathcal{P}_{d}(\mathbf{n})$ such that $\mathcal{P}_{d}(\mathbf{n}) \sigma \mathcal{P}_{d}(\mathbf{n}) \subset \mathcal{P}_{d}(\mathbf{n}) \pi \mathcal{P}_{d}(\mathbf{n}), \Psi(\sigma)=\mathbf{v}$ and $\Psi(\pi)=\mathbf{w}$.

On the other hand, from the last paragraph of the proof of Proposition 25, it follows that the poset $\left(\mathcal{P}_{d}(\mathbf{n}) / \mathcal{J}, \rightsquigarrow\right)$ is a graded poset. Now, applying the argument from the third paragraph of the proof of Proposition 25 once more and counting modulo $d$, one checks that the covering relations in $\left(\mathcal{P}_{d}(\mathbf{n}) / \mathcal{J}, \rightsquigarrow\right)$ and $(I(n \mathbf{e}(1)), \prec)$ match precisely via $\Psi$. The claim follows.

\section{Enumeration of $\mathcal{J}$-classes for arbitrary $d$}

\subsection{Enumeration via $d$-part partitions}

The proof of Proposition 25 gives a way to write a formula for $C_{n}^{(d)}$ in the general case. Let $d \in \mathbb{N}$ and $n \in \mathbb{Z}_{\geq 0}$. Denote by $P_{n}^{(d)}$ the number of partitions of $n$ with at most $d$ parts. By taking the dual partition, we get the usual fact that $P_{n}^{(d)}$ also equals the number of partitions of $n$ in which each part does not exceed $d$. For simplicity, we set $P_{n}^{(d)}=0$ when $n<0$.

Theorem 28. We have $C_{n}^{(d)}=P_{n}^{(d)}+P_{n-d}^{(d)}+P_{n-2 d}^{(d)}+P_{n-3 d}^{(d)}+\ldots$

Proof. To prove this claim, we analyse the proof of Proposition 25. According to the latter proof, $C_{n}^{(d)}$ enumerates canonical elements in $\mathcal{P}_{d}(\mathbf{n})$. Let $\sigma$ be a canonical element. Let $\sigma_{1}, \sigma_{2}, \ldots, \sigma_{k}$ be the list of all parts of $\sigma$ contained in $\underline{n}$ (note that $k$ might be zero). Then, each of these parts has cardinality $d$, and we may consider the set

$$
\underline{n}^{\sigma}:=\underline{n} \backslash\left(\sigma_{1} \cup \sigma_{2} \cup \cdots \cup \sigma_{k}\right)
$$

which thus has cardinality $n-k d$.

Cardinalities of intersections of all propagating parts of $\sigma$ with $\underline{n}^{\sigma}$ determine a partition of $n-k d$ in which each part does not exceed $\bar{d}$. It is straightforward that this gives a bijection between the set of all canonical elements in $\mathcal{P}_{d}(\mathbf{n})$ with $2 k$ non-propagating parts and all partitions of $n-k d$ for which each part does not exceed $d$. The claim follows. 
Corollary 29. For $d \geq 1$, we have

$$
\sum_{n \geq 1} C_{n}^{(d)} t^{n}=\frac{1}{\left(1-t^{d}\right) \cdot(1-t)\left(1-t^{2}\right)\left(1-t^{3}\right) \ldots\left(1-t^{d}\right)} .
$$

Proof. This follows by combining the usual equality

$$
\sum_{n \geq 1} P_{n}^{(d)} t^{n}=\frac{1}{(1-t)\left(1-t^{2}\right)\left(1-t^{3}\right) \ldots\left(1-t^{d}\right)}
$$

with the statement of Theorem 28 .

Remark 30. It is easy to check that for $d=3$, we indeed have the equality

$$
\frac{1+t^{2}+t^{3}+t^{5}}{(1-t)\left(1-t^{3}\right)\left(1-t^{4}\right)\left(1-t^{6}\right)}=\frac{1}{(1-t)\left(1-t^{2}\right)\left(1-t^{3}\right)^{2}} \text {. }
$$

Here, the left-hand side is the original generating function for $A 028289$.

Remark 31. The poset $\Pi^{(d)}$ of partitions with at most $d$ parts can be defined using the same approach as we used to define $\Lambda_{d}$. The assertion of Theorem 28 can then be interpreted as a bijection between certain (co)ideals in $\Pi^{(d)}$ and $\Lambda_{d}$. Such a bijection admits a direct combinatorial construction.

\subsection{Examples for $d=4$ and $d=5$}

The sequence $C_{n}^{(4)}$ starts as follows:

$$
1,1,2,3,6,7,11,14,21,25, \ldots
$$

The sequence $C_{n}^{(5)}$ starts as follows:

$$
1,1,2,3,5,8,11,15,21, \ldots
$$

We note that none of the sequences $C_{n}^{(d)}$ for $d \geq 4$ appeared on [19] before. However, as noted, they are simple cumulative sums of classical sequences.

\subsection{Relation to partition function}

Recall the classical partition function $P(n)$ which gives, for $n \in \mathbb{Z}_{\geq 0}$, the number of partitions of $n$, see the sequence $A 000041$ in [19]. One general observation for the numbers $C_{n, h}^{(d)}$ is the following:

Proposition 32. If $n-h<d$ and $2(n-h)<n$, then $C_{n, h}^{(d)}=P(n-h)$.

Proof. To prove the assertion, we construct a bijective map between $I(n \mathbf{e}(1)) \cap$ $\Lambda_{d}^{(h)}$ and the set of all partitions of $n-h$.

For $\mathbf{v} \in \Lambda_{d}$, set $\alpha(\mathbf{v})=v_{2}+2 v_{3}+3 v_{4}+\ldots$ For $i, j \in\{1,2, \ldots, d\}$ such that $i+j<d$, we have $(i+j-1)-(i-1)-(j-1)=1$. Therefore, for such values of $i$ and $j$ and for any $\mathbf{v}, \mathbf{w} \in \Lambda_{d}$, we have $\alpha(\mathbf{v})=\alpha(\mathbf{w})+1$ provided that

$$
\mathbf{v}=\mathbf{w}+\mathbf{e}(i+j)-\mathbf{e}(i)-\mathbf{e}(j)
$$

Note that $\mathbf{e}(i+j)-\mathbf{e}(i)-\mathbf{e}(j) \in X_{d}$.

Assume now that $n-h<d$ and $\mathbf{v} \in I(n \mathbf{e}(1))$ is of height $h<n$. Then, $\mathbf{v}$ is obtained from $n \mathbf{e}(1)$ by adding $n-h$ vectors from $X_{d}$ of the form 
$\mathbf{e}(i+j)-\mathbf{e}(i)-\mathbf{e}(j)$, for some $i$ and $j$ as above. Indeed, let $s>1$ be the smallest index such that $v_{i} \neq 0$ (which exists as $h<n$ and $n-h<d$ ). Then, the vector $\mathbf{v}$ is obtained from the vector

$$
\mathbf{w}=\left(v_{1}+1, v_{2}, \ldots, v_{s-2}, v_{s-1}+1, v_{s}-1, v_{s+1}, \ldots, v_{d}\right)
$$

by adding $\mathbf{e}((s-1)+1)-\mathbf{e}(s-1)-\mathbf{e}(1)$ (here $w_{1}=v_{1}+2$ if $\left.s=2\right)$. Applying a similar procedure to $\mathbf{w}$ and proceeding inductively, we get the claim. This implies that

$$
\Upsilon(\mathbf{v}):=\left(v_{2}+v_{3}+v_{4}+\ldots, v_{3}+v_{4}+\ldots, \ldots\right)
$$

is a partition of $n-h$. Since $n-h$ is fixed and $\mathbf{v}$ is, clearly, recoverable from $\Upsilon(\mathbf{v})$, the map $\Upsilon$ from $I(n \mathbf{e}(1)) \cap \Lambda_{d}^{(h)}$ to the set of all partitions of $n-h$ is injective.

To prove surjectivity of $\Upsilon$, assume that $n-h=x_{2}+2 x_{3}+3 x_{4}+\ldots$, for some non-negative $x_{2}, x_{3}, \ldots$ We proceed by induction on $n-h$. If $n-h=$ 0 , surjectivity of our map is obvious. To prove the induction step, we write $k=i+j$, for some $1 \leq i, j \leq k-1$, and consider the partition of $n-h-1$ given by decreasing $x_{k}$ by 1 , increasing $x_{i}$ by 1 and increasing $x_{j}$ by 1 (if $i=j$, the outcome is that $x_{i}$ is increased by 2). From the combination of the inductive assumption and the condition $2(n-h)<n$, it follows that the resulting partition of $n-h-1$ is in the image of our map. Applying the definition of $\prec$, it follows that the original partition of $n-h$ is also in the image of our map. This completes the proof.

Problem 33. Find a closed formula for $C_{n, h}^{(d)}$ for all $d, n, h$.

\section{Acknowledgements}

An essential part of the research was done during the visit of the third author to Leeds in October 2014, which was supported by EPSRC under grant $\mathrm{EP} / \mathrm{I} 038683 / 1$. The paper was completed during the stay of the second and the third authors at the Institute Mittag-Leffler in the spring of 2015. The financial support and hospitality of both the University of Leeds and the Institute Mittag-Leffler are gratefully acknowledged. The first author is supported by the KRG via the HCDP Scholarship program. For the third author, the research was partially supported by the Royal Swedish Academy of Sciences, Knut and Alice Wallenbergs Stiftelse and the Swedish Research Council. We thank M. Kosuda for helpful discussions. We thank the referee for helpful comments and for pointing out several inaccuracies in the original version of the paper.

Funding Open Access funding provided by Uppsala University.

Open Access. This article is licensed under a Creative Commons Attribution 4.0 International License, which permits use, sharing, adaptation, distribution and reproduction in any medium or format, as long as you give appropriate credit to the original author(s) and the source, provide a link to the Creative Commons licence, 
and indicate if changes were made. The images or other third party material in this article are included in the article's Creative Commons licence, unless indicated otherwise in a credit line to the material. If material is not included in the article's Creative Commons licence and your intended use is not permitted by statutory regulation or exceeds the permitted use, you will need to obtain permission directly from the copyright holder. To view a copy of this licence, visit http://creativecommons. org/licenses/by/4.0/.

Publisher's Note Springer Nature remains neutral with regard to jurisdictional claims in published maps and institutional affiliations.

\section{References}

[1] G. Birkhoff. Lattice Theory. American Mathematical Society Colloquium Publications 25, revised edition. American Mathematical Society, New York, N. Y., 1948.

[2] A. Björner. Shellable and Cohen-Macaulay partially ordered sets. Trans. Amer. Math. Soc. 260 (1980), no. 1, 159-183.

[3] B. N. Cyvin, L. J. Brunvoll and S. J. Cyvin. Enumeration of Conjugated Hydrocarbons: Hollow Hexagons Revisited. Structural Chemistry 6 (1995), No. 2, $85-88$.

[4] S. Cyvin, J. Brunvoll, B. Cyvin, J. Bergan, and E. Brendsdal. The Simplest Coronoids: Hollow Hexagons. Structural Chemistry 2 (1991), No. 6, 555-566.

[5] O. Ganyushkin, V. Mazorchuk. Classical finite transformation semigroups. An introduction. Algebra and Applications, 9. Springer-Verlag London, Ltd., London, 2009.

[6] O. Ganyushkin, V. Mazorchuk and B. Steinberg. On the irreducible representations of a finite semigroup, Proc. Amer. Math. Soc. 137 (2009), 3585-3592.

[7] N. Ham. The planar modular partition monoid. PhD Thesis, University of Tasmania, 2015.

[8] V. Jones. The Potts model and the symmetric group. In: Subfactors: Proceedings of the Taniguchi Symposium on Operator Algebras (Kyuzeso, 1993), River Edge, NJ, World Sci. Publishing, 1994, pp. 259-267.

[9] M. Kosuda. Characterization for the party algebra, Ryukyu Math. J. 13 (2000), $7-22$.

[10] M. Kosuda. Irreducible representations of the party algebra. Osaka J. Math. 43 (2006), no. 2, 431-474.

[11] M. Kosuda. Party algebra of type $B$ and construction of its irreducible representations. Manuscript available via google.

[12] P. P. Martin. Potts models and related problems in statistical mechanics. World Scientific, Singapore, 1991. 
[13] P. P. Martin. Temperley-Lieb algebras for non-planar statistical mechanics the partition algebra construction. Journal of Knot Theory and its Ramifications 3 (1994), no. 1, 51-82.

[14] P. P. Martin. The structure of the partition algebras. J. Algebra 183 (1996), no. 2, 319-358.

[15] V. Mazorchuk. On the structure of Brauer semigroup and its partial analogue. Problems in Algebra 13 (1998), Gomel: University Press, 29-45.

[16] V. Mazorchuk. Endomorphisms of $\mathfrak{B}_{n}, \mathcal{P} \mathfrak{B}_{n}$ and $\mathfrak{C}_{n}$. Comm. Algebra 30 (2002), no. $7,3489-3513$.

[17] R. Orellana. On partition algebras for complex reflection groups. J. Algebra 313 (2007), no. 2, 590-616.

[18] R. Orellana. On the algebraic decomposition of a centralizer algebra of the hyperoctahedral group. Algebraic structures and their representations, 345-357, Contemp. Math., 376, Amer. Math. Soc., Providence, RI, 2005.

[19] N. Sloane. The On-Line Encyclopedia of Integer Sequences. https://oeis.org/

[20] G. Polya and R. Read. Combinatorial enumeration of groups, graphs and chemical compounds. Springer, 1987.

[21] L. Rigal, P. Zadunaisky. Twisted semigroup algebras. Algebr. Represent. Theory 18 (2015), no. 5, 1155-1186.

[22] K. Tanabe. On the centralizer algebra of the unitary reflection group $G(m, p, n)$. Nagoya Math. J. 148 (1997), 113-126.

[23] G. Ziegler. On the poset of partitions of an integer. J. Combin. Theory Ser. A 42 (1986), no. 2, 215-222.

Chwas Ahmed and Paul Martin

Department of Pure Mathematics

University of Leeds

Leeds LS2 9JT

UK

e-mail: ppmartin@maths.leeds.ac.uk

Present Address

Chwas Ahmed

Department of Mathematics, College of Science

University of Sulaimani

Sulaimani

Iraq

e-mail: chwas.ahmed@univsul.edu.iq 
Volodymyr Mazorchuk

Department of Mathematics

Uppsala University

Box 480, SE-75106, Uppsala

Sweden

e-mail: mazor@math.uu.se

Received: 12 July 2015.

Accepted: 20 December 2019. 